\title{
Radioluminescent Nanoparticles for Radiation-Controlled Release of Drugs
}

Rahul Misra, ${ }^{1}$ Kaustabh Sarkar, ${ }^{1}$ Jaewon Lee, ${ }^{1}$ Vincenzo J. Pizzuti, ${ }^{1}$ Deborah S. Lee, ${ }^{1}$ Melanie P. Currie, ${ }^{2}$ Sandra E. Torregrosa-Allen, ${ }^{2}$ David E. Long, ${ }^{3}$ Gregory A. Durm, ${ }^{4}$ Mark P. Langer, ${ }^{3}$ Bennett D. Elzey, ${ }^{2}$ and You-Yeon Won ${ }^{1,2, *}$ yywon@ecn.purdue.edu

${ }^{1}$ Davidson School of Chemical Engineering, Purdue University, West Lafayette, Indiana 47907, United States

${ }^{2}$ Purdue University Center for Cancer Research, Purdue University, West Lafayette, Indiana 47907, United States

${ }^{3}$ Department of Radiation Oncology, Indiana University School of Medicine, Indianapolis, Indiana 46202, United States

${ }^{4}$ Department of Medicine, Indiana University School of Medicine, Indianapolis, Indiana 46202, United States

*Corresponding author.

This is the author's manuscript of the article published in final edited form as:

Misra, R., Sarkar, K., Lee, J., Pizzuti, V. J., Lee, D. S., Currie, M. P., .. Won, Y.-Y. (2019). Radioluminescent nanoparticles for radiation-controlled release of drugs. Journal of Controlled Release. https://doi.org/10.1016/j.jconrel.2019.04.033 


\section{ABSTRACT}

The present work demonstrates a novel concept for intratumoral chemo-radio combination therapy for locally advanced solid tumors. For some locally advanced tumors, chemoradiation is currently standard of care. This combination treatment can cause acute and long term toxicity that can limit its use in older patients or those with multiple medical comorbidities. Intratumoral chemotherapy has the potential to address the problem of systemic toxicity that conventional chemotherapy suffers, and may, in our view, be a better strategy for treating certain locally advanced tumors. The present study proposes how intratumoral chemoradiation can be best implemented. The enabling concept is the use of a new chemotherapeutic formulation in which chemotherapy drugs (e.g., paclitaxel (PTX)) are coencapsulated with radioluminecsnt nanoparticles (e.g., $\mathrm{CaWO}_{4}$ (CWO) nanoparticles (NPs)) within protective capsules formed by biocompatible/biodegradable polymers (e.g., poly(ethylene glycol)-poly(lactic acid) or PEG-PLA). This drug-loaded polymer-encapsulated radioluminescent nanoparticle system can be locally injected in solution form into the patient's tumor before the patient receives normal radiotherapy (e.g., $30-40$ fractions of 2 - 3 Gy daily X-ray dose delivered over several weeks for locally advanced head and neck tumors). Under Xray irradiation, the radioluminescent nanoparticles produce UV-A light that has a radiosensitizing effect. These co-encapsulated radioluminescent nanoparticles also enable radiationtriggered release of chemo drugs from the polymer coating layer. The non-toxic nature (absence of dark toxicity) of this drug-loaded polymer-encapsulated radioluminescent nanoparticle ("PEG-PLA/CWO/PTX") formulation was confirmed by the MTT assay in cancer cell cultures. A clonogenic cell survival assay confirmed that these drug-loaded polymer-encapsulated radioluminescent nanoparticles significantly enhance the cancer cell killing effect of radiation therapy. In vivo study validated the efficacy of PEG-PLA/CWO/PTX-based intratumoral chemoradio therapy in mouse tumor xenografts (in terms of tumor response and mouse survival). Results of a small-scale NP biodistribution (BD) study demonstrate that PEG-PLA/CWO/PTX NPs remained at the tumor sites for a long period of time (>1 month) following direct intratumoral administration. A multi-compartmental pharmacokinetic model (with rate constants estimated from in vitro experiments) predicts that this radiation-controlled drug release technology enables significant improvements in the level and duration of drug availability within the tumor (throughout the typical length of radiation treatment, i.e., $>1$ month) over 
conventional delivery systems (e.g., PEG-PLA micelles with no co-encapsulated $\mathrm{CaWO}_{4}$, or an organic liquid, e.g., a 50:50 mixture of Cremophor EL and ethanol, as in Taxol), while it is capable of maintaining the systemic level of the chemo drug far below the toxic threshold limit over the entire treatment period. This technology thus has the potential to offer a new therapeutic option that has not previously been available for patients excluded from conventional chemoradiation protocols.

KEYWORDS: Radioluminescent nanoparticles; radiotherapy; chemotherapy; chemoradiation; radiation-controlled drug release; PEG-PLA; paclitaxel; head and neck squamous cell carcinoma 


\section{INTRODUCTION}

More than 51,540 new cases of head and neck cancer were diagnosed within USA in 2018. Out of these cases, 10,030 deaths were reported [1]. Most patients at the time of diagnosis are at a advanced stage, with $40 \%$ of the patients with regional lymph node involvement and $10 \%$ with distant metastasis [2]. Currently, the treatment options for patients with locally advanced head and neck carcinomas consist of surgery followed by appropriate adjuvant therapy (with or without radiation and with or without chemotherapy), concurrent chemoradiation, induction chemotherapy followed by local therapy, or radiation alone [1].

Oropharyngeal and palliative surgeries are difficult in terms of technical approach. Radiation therapy given after surgery is beneficial for a few months; however, there are still high chances of recurrence with five-year survival rates of only $20-40 \%$ [3-5]. Chemotherapy, in general, is better tolerated than surgery followed by radiation, as drug dose and route of administration can be tailored depending on the patient's condition. However, chemotherapy alone for head and neck cancers has not been shown to be curative, and a local therapy (surgery or radiation) is needed. Concurrent chemoradiation has been shown to be more effective than radiation alone [6]. The adverse effects of chemotherapy are well documented. In conventional chemotherapy, chemotherapeutic drugs are administered intravenously into the bloodstream periodically (alternative days, weeks, etc.) over "cycles". In this process, the drug concentration in the blood varies with time between a maximum concentration (which may represent a toxic level) and a minimum value (which is typically below the limit at which the drug becomes ineffective). In each cycle, the drug concentration inside the body reaches a toxic level (referred as a "peak") and then decreases after about $4-12$ hours $[5,7,8]$. To maintain the level of the chemo drug in the therapeutic range, the drug is administered in multiple cycles, each with a dose higher than the therapeutic limit. Consequently, the drugs at such toxic level not only kill cancer cells but also affect the healthy tissues of the body. There are substantial short and longterm side effects associated with systemic chemotherapy. This is due to the uncontrolled concentration of the drug in the body during the peaks of the chemotherapy cycles with direct administration of the drug. Short-term side effects include the toxic effects observed during chemotherapy regimen, while long-term side effects include later complications of the treatment arising after the completion of adjuvant chemotherapy. Depending on the individual, specific 
agent used, amount of dose, duration of treatment in the adjuvant regimen, etc., the intensities of these side effects vary [9].

Sequential chemotherapy administration, in the form of induction chemotherapy followed by radiotherapy or concurrent chemoradiation, has been used for head and neck cancer [10]. A significant improvement in disease-free and locoregional recurrence-free survival rate has been observed; the survival rate has been reported to be around $50-60 \%$ at 5 years $[11,12]$. However, symptoms of grade 2, 3 and even grade 4 toxicity (such as rash, fatigue, neuropathy, anemia, alopecia, myelosuppression, neutropenia, mucositis, immunosuppression, xerostomia, hypothyroidism, diarrhea, and constipation) have been observed in different clinical studies with systemic chemotherapy [13-16]. A recent study has reported certain changes in gut microbiota leading to clostridium difficile infection (CDI) in head and neck cancer patients who received systemic paclitaxel chemotherapy followed by radiation [17]. In addition, the multiple patient exclusion criteria limit the applicability of the current chemo-radio protocol to within a particular domain of patients. For example, patients above 70 years of age, patients associated with certain other medical conditions such as uncontrolled hypertension, ischemic heart disease, human immunodeficiency virus infection, diabetes mellitus, mental disability, chronic liver disease, or pulmonary tuberculosis, patients with prior or synchronous malignancy, previous radiotherapy or chemotherapy, pregnancy or current lactation, patients with sickle cell disease, and patients with distant metastasis may be excluded from the present treatment regimen $[18,19]$.

There has been a steady growth in research on intratumoral chemotherapy during the past few decades as an alternative to the conventional systemic delivery approach for patients with unresectable lesions [20,21]. The first known clinical study of intratumoral chemotherapy was performed in 1958 using triethylene phosphoramide and oxapentamethylene, which resulted in significant tumor regression for advanced cancers of the breast, liver and ovaries [22]. Since the 1990s, there has been a steady increase in the development of controlled release drug delivery vehicles for intratumoral treatment due to its advantages over systemic therapies. Several formulations based on polymers, liposomes and nanoparticles have been studied as implantable controlled release depots, particularly, for hydrophobic drugs, such as cisplatin and paclitaxel $[20,21,23,24]$. One such formulation, Gliadel ${ }^{\mathrm{TM}}$ wafers (polymer-based implants) are currently used clinically to treat an aggressive form of brain cancer [25]. Alternatively, recently developed 
hafnium oxide nanoparticle radio-sensitizers are delivered via intratumoral injection for advanced head and neck cancers, and are currently in Phase II clinical trials [26]. Safety and efficacy of locally delivered chemotherapies (carmustine, carboplatin, and camptothecin) have been tested in combination with concurrent radiation therapy for treating metastatic brain [27] and breast [28] tumors; these studies demonstrated that this local chemo-radiation combination is safe and effective in prolonging survival in mouse models of metastatic brain and breast cancers. Intratumoral administration of chemotherapeutic drugs can provide localization of the drugs within the tumor and can prevent exposure of the non-target organs to such drugs, resulting in reduced toxicity and better efficacy. It can be a promising approach not only for the treatment of locally advanced solid tumors but also for malignant gliomas in adjunct therapy [18, 19, 29, 30]. Moreover, it has the potential to be an effective loco-regional therapy, especially for the patients who fall under the exclusion criteria of the current systemic chemo-radio therapy.

Polymeric carrier systems are known for their biocompatible nature and ability to sustain delivery of drugs [31]. The poly(ethylene glycol-b-D,L-lactic acid)(PEG-PLA)-based paclitaxel (PTX) formulation, commercially known as Genexol-PM (or Cynviloq ${ }^{\mathrm{TM}}$ in the United States), is an FDA-equivalent-approved example. Intratumoral pharmacokinetic studies have shown that the polymeric formulation can confine the drug (paclitaxel) within the tumor two times longer than the paclitaxel administered in the form of an organic dispersion [30].

Polymeric formulations release the encapsulated drug in a sustained manner. However, there is still need for a better means to control the drug release rate in order to maintain the concentration of the drug inside the tumor within the therapeutically effective range for an extended period of time. This need is addressed by the radiation-controlled chemo drug release system disclosed in the present article (Scheme 1). In this work, a novel chemo-radio combination therapy protocol enabled by PTX-loaded PEG-PLA-encapsulated $\mathrm{CaWO}_{4}(\mathrm{CWO})$ nanoparticles (NPs) is demonstrated. Our laboratory has been pioneering the use of CWO NPs to enhance cancer radiotherapy. $\mathrm{CaWO}_{4}$ is a naturally abundant radio-luminescent material ("scintillator") that has been commonly used for X-ray imaging [32]. $\mathrm{CaWO}_{4}$ has a solubility product constant of $\mathrm{K}_{\mathrm{sp}} \approx 4.9 \times 10^{-10}$ (at a neutral $\mathrm{pH}$ at $298 \mathrm{~K}$ ) and therefore can only liberate non-toxic amounts of $\mathrm{WO}^{-2}$ ions [33]. $\mathrm{WO}^{-2}$ ions are typically rapidly cleared from the body by the kidneys [34]. $\mathrm{CaWO}_{4}$ is known to have no carcinogenic properties [35]. CWO NPs can 
effectively be encapsulated within self-assemblies of block copolymers [35]. Recently, we have shown that PEG-PLA-encapsulated $\mathrm{CaWO}_{4}$ nanoparticles (PEG-PLA/CWO NPs) are promising as a contrast agent for X-ray imaging [36] and also as a radio-sensitizer for cancer radiation therapy [33]. The work described in the present manuscript is an extension of these previous studies aimed at developing CWO NP-based radio-sensitizers. Specifically, this work investigates how the incorporation of the additional therapeutic component, PTX, affects the radio-sensitization ability of the PEG-PLA/CWO NP system. PTX-loaded PEG-PLA/CWO NPs (PEG-PLA/CWO/PTX NPs) can be directly injected into solid tumor. An in vivo BD study confirms that following intratumoral administration, these NPs stay within the tumor for a long time (for at least one month). During radiotherapy, X-rays $/ \gamma$ rays trigger the release of encapsulated PTX from the polymer coating layer; controlled experiments suggest that the mechanism of radiation-induced PTX release involves two processes: partitioning/accumulation of PTX in the vicinity of the $\mathrm{CaWO}_{4}$ core (causing very slow release of PTX in the absence of radiation), and (2) photolytic degradation of PLA (triggering rapid PTX release under X-ray or UV-A irradiation). PTX is a standard drug in first-line chemotherapy for head and neck cancer [37]. Concurrent PEG-PLA/CWO/PTX NPs produce synergistic interactions with $\mathrm{X}$-ray/ $\gamma$ ray radiation due to the radiosensitization effects of PTX $[11,31,38$, 39] and UV-A light generated by CWO NPs under X-ray/ $\gamma$ ray irradiation [40]. In vitro clonogenic and in vivo tumor suppression/mouse survival assays validate that PEGPLA/CWO/PTX NPs are indeed capable of inducing a significant enhancement of the tumor suppressive effect of X-rays. A multicompartmental PK model based on experimental rate constants predicts that the radiation-controlled drug release mechanism enables to maintain the drug concentration within the tumor at the therapeutic level for an extended period of time, while it also enables to maintain the drug concentration in the systemic circulation well below the toxic threshold; similar PK simulations performed for other PTX formulations (PTX-loaded PEG-PLA micelles with no co-encapsulated $\mathrm{CaWO}_{4}$, and PTX dispersed in Cremophor EL/ethanol (Taxol)) suggest that these conventional PTX formulations are less desirable for use in IT chemoradiotherapy. The present radiation-controlled chemo drug release technology is expected to enable intratumoral chemo-radiotherapy treatment for frail patients who do not meet the inclusion criteria for standard chemo-radio treatment guidelines. Intratumoral chemo- 
radiotherapy is expected to offer a treatment option that delays the progression or relapse of the disease without compromising the quality of life for patients.
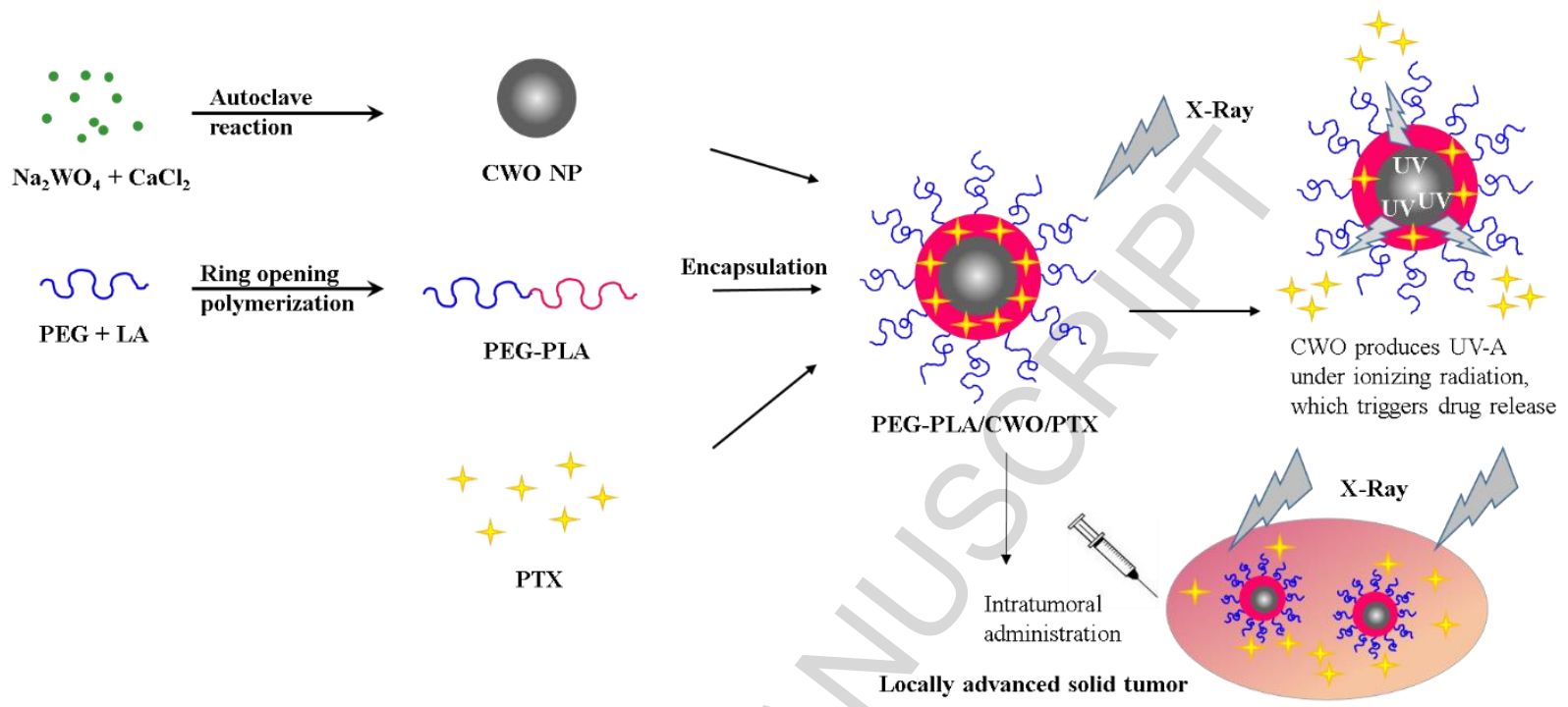

Scheme 1. Schematic illustration of the preparation of poly(ethylene glycol-b-lactic acid)(PEG-PLA)-encapsulated $\mathrm{CaWO}_{4}$ (CWO) nanoparticles (NPs) loaded with chemotherapeutic drugs, paclitaxel (PTX), and the release of PTX from PEG-PLA/CWO NPs upon exposure to X-rays. CWO NPs are radio-luminescent; they produce longwavelength UV light in the "UV-A" (320 - $400 \mathrm{~nm})$ range under X-ray irradiation [35]. CWO NPs are coated with PEG-PLA block copolymers. PEG chains are hydrophilic and stay in the aqueous phase. The CWO NP core is coated with hydrophobic PLA chains. PTX is encapsulated within the hydrophobic PLA layer. Under X-ray irradiation, UV-A is generated by CWO NPs, and X-rays and UV-A light cause the release of PTX from the PLA layer into the aqueous surrounding. Intratumorally administered PEG-PLA/CWO/PTX NPs release PTX in tumor during radiation treatments. The PTX release rate is controlled by radiation dose.

\section{MATERIALS \& METHODS}

\subsection{Synthesis and characterizations of PEG-PLA block copolymers and CWO NPs}

The PEG-PLA block copolymer material used in this study $\left(\mathrm{M}_{\mathrm{n}}, \mathrm{PEG}=5,000 \mathrm{Da}, \mathrm{M}_{\mathrm{n}}, \mathrm{PLA}=\right.$ 5,000 Da) was synthesized via 1,8-diazabicyclo[5.4.0]undec-7-ene(DBU)-catalyzed ring-opening polymerization of racemic lactide from a monomethoxy/monohydroxy-terminated PEG macroinitiator, as has been described in a previous publication [40]. Conversion to the PEG-PLA product was confirmed by ${ }^{1}$ H-NMR (Supplementary Material (SM) Figure S1). Primary CWO NPs of a monodisperse diameter $(10 \mathrm{~nm})$ were synthesized using a microemulsion method as also described in [40]. 


\subsection{Preparation and characterizations of PTX-loaded PEG-PLA-encapsulated CWO NPs}

Our laboratory has developed a procedure for preparation of PEG-PLA-encapsulated CWO NPs [36, 40]. This procedure was adopted to prepare PTX-loaded PEG-PLA-encapsulated CWO NPs (having a mean hydrodynamic diameter of about $50 \mathrm{~nm}$ ). PTX was purchased from either Samyang Biopharmaceuticals (used in all studies reported in this article except for the in vivo CWO NP BD study described in Section 2.11) or Biotang (used in the CWO BD study). $300 \mathrm{mg}$ of PEG-PLA block copolymers (BCP) and $30 \mathrm{mg}$ of PTX were dissolved in $3.8 \mathrm{~g}$ of dimethylformamide (DMF, > 99.9\% purity, Sigma-Aldrich). $0.5 \mathrm{mg}$ of CWO NPs (10 nm diameter) were dispersed in $2.1 \mathrm{~g}$ of Milli-Q-purified water. These two solutions were mixed together rapidly under simultaneous high-speed mechanical stirring (15,000 rpm) and ultrasonication for 30 minutes. The resultant mixture was centrifuged at 5,000 rpm (equivalent to $3214 \times \mathrm{g}$, Eppendorf $5804(\mathrm{~F}-34-6-38$, radius of rotor $=11.5 \mathrm{~cm}))$ for 10 minutes. The supernatant containing unencapsulated PTX, excess PEG-PLA and DMF was removed. The precipitate was dried under vacuum oven overnight. Assuming a hydrodynamic diameter of 66 nm for PEG-PLA/CWO/PTX NPs in the 3.8:2.1 DMF/water mixture (based on data shown in SM Figure S2) and a composition of PEG:PLA:CWO:PTX: water $=0.65: 0.65: 1.0: 0.12: 0.65$ by weight (see the figure caption for Figure 5) within the hydrodynamic volume of a PEGPLA/CWO/PTX NP, the average mass density of a PEG-PLA/CWO/PTX NP within its hydrodynamic volume is estimated to be $1.54 \mathrm{~g} / \mathrm{cc}$, which gives an estimate of $2.28 \times 10^{3} \mathrm{~s}$ for the time it takes for a PEG-PLA/CWO/PTX NP to sediment a distance of $1.0 \mathrm{~cm}$ (the height of liquid in the centrifugation tube) at $3214 \times \mathrm{g}$ centrifugal acceleration using the equation, $\tau \approx$ $91 \eta /\left(2 R^{2} \Delta \rho g\right)$, where $\tau$ is the sedimentation time, 1 is the sedimentation distance, $\eta$ is the viscosity of the medium, $\mathrm{R}$ is the radius of the NP, $\Delta \rho$ is the density difference between the NP and medium, and $\mathrm{g}$ is the gravitational acceleration constant; therefore, during the 10-minute centrifugation time, about $26 \%$ of PEG-PLA/CWO/PTX NPs will have sedimented to the bottom of the vial. Similarly, assuming the same hydrodynamic diameter $(\approx 66 \mathrm{~nm}$, consistent with Ref. [41]) for PEG-PLA/PTX NPs in the DMF/water mixture and a composition of PEG:PLA:PTX:water $=0.65: 0.65: 0.12: 0.65$ by weight within the hydrodynamic volume of a PEG-PLA/PTX NP, the average mass density of a PEG-PLA/PTX NP within its hydrodynamic volume is estimated to be $1.12 \mathrm{~g} / \mathrm{cc}$, which gives an estimate of $9.36 \times 10^{3} \mathrm{~s}$ for the time it takes for a PEG-PLA/PTX NP to sediment a distance of $1.0 \mathrm{~cm}$ at $3214 \times \mathrm{g}$ centrifugal acceleration; 
therefore, during the 10-minute centrifugation time, about $6.4 \%$ of PEG-PLA/PTX NPs will have sedimented to the bottom of the vial (94\% of non-CWO-containing PEG-PLA/PTX NPs will remain in the supernatant). Therefore, it is reasonable to expect that the above centrifugation step removes most of the excess PEG-PLA and PTX.

After re-dispersing PTX-loaded PEG-PLA-encapsulated CWO NPS in PBS at a NP concentration of $0.25 \mathrm{mg}$ CWO per $\mathrm{mL}$ and filtering the solution with a $0.22 \mu \mathrm{m}$ PVDF filter, their mean hydrodynamic size was determined by dynamic light scattering (DLS) using a Brookhaven Instruments ZetaPALS instrument; data presented in SM Figure S2; the mean hydrodynamic diameters of PEG-PLA/CWO/PTX and PEG-PLA/CWO NPs in Milli-Q water were 65.6 and $40.8 \mathrm{~nm}$, respectively (Figure S2).. In DLS characterization, the filtration step was necessary to remove a very small amount of large aggregates. In all other studies discussed in this article, unfiltered samples were used, because filtration did not influence results; of note, PTX release kinetics is expected to be primarily controlled by the thickness of the PLA layer, which in turn is primarily controlled by the molecular weight of the PLA block (currently, study is underway to investigate how PEG-PLA molecular weight characteristics affect PTX release kinetics). The morphologies of these nanoparticles were also examined with transmission electron microscopy (TEM) using $2 \%$ uranyl acetate as a negative staining agent (Figure 1).
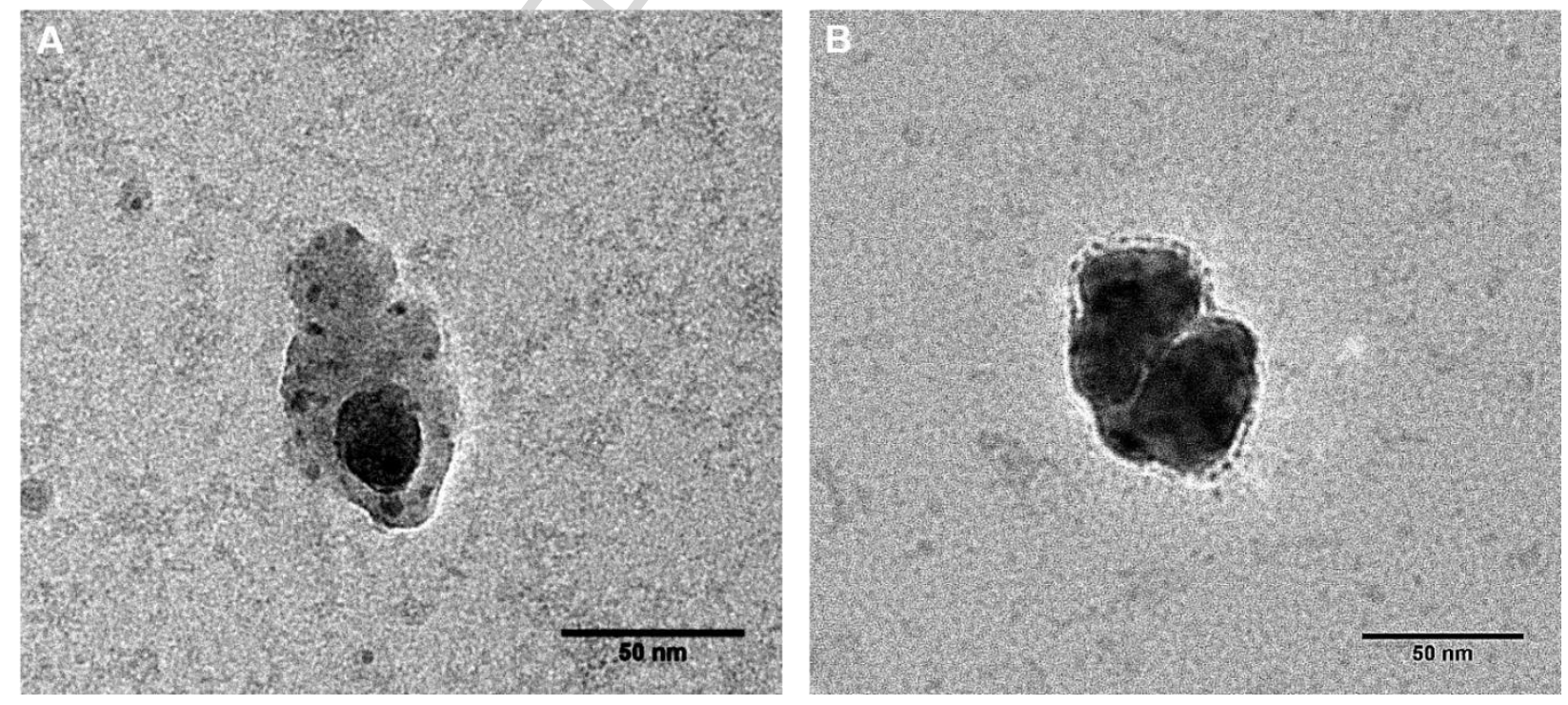

Figure 1. Representative TEM micrographs of (A) PEG-PLA/CWO NPs, and (B) PEG-PLA/CWO/PTX NPs. Dried specimens were stained with uranyl acetate. Scale bars are $50 \mathrm{~nm}$ in both images. 


\subsection{In vitro drug release kinetics}

To measure the rate of PTX release from PTX-loaded PEG-PLA-coated CWO NPs, the dried pellet obtained from the previous step was re-dispersed in PBS at a CWO concentration of $0.25 \mathrm{mg} / \mathrm{mL}$, and the solution was placed in a dialysis tube (50 kDa MWCO). The dialysis tube was sealed at both ends, submerged in $50 \mathrm{~mL}$ of PBS, and kept under mild stirring using a magnetic stirring bar. PTX release measurements were performed on four samples: (1) X-rayirradiated vs. (2) non-X-ray-irradiated PTX-loaded PEG-PLA-encapsulated CWO NPs, and (3) X-ray-irradiated vs. (4) non-X-ray-irradiated PTX-loaded PEG-PLA micelles (with no coencapsulated CWO NPs). X-ray irradiation was performed at 7 Gy on Day 2 following resuspension in PBS. At regular intervals, $50 \mathrm{~mL}$ of the dialysis medium was taken for measurement of PTX concentration; each time the same volume of blank PBS was added to the medium to compensate for the volume loss. PTX was collected from the dialysis sample by liquid-liquid extraction as described below. $30 \mathrm{~mL}$ of dichloromethane (DCM, > 99.9\% purity, Sigma-Aldrich) was added to $50 \mathrm{~mL}$ of the dialysis sample. This mixture was vigorously shaken for a few minutes, and then kept undisturbed for 30 minutes until two distinct liquid layers were formed. The bottom DCM solution was carefully collected and was dried under vacuum oven overnight. The dried substance (PTX) was dispersed in $2 \mathrm{~mL}$ of a 1:1 by volume mixture of water and acetonitrile (HPLC solvent) and analyzed by HPLC for determination of the PTX concentration as follows. A C18 column with dimensions $4 \times 125 \mathrm{~mm}$ (Agilent 1100 Hypersil, 5 $\mu \mathrm{M})$ was used as the stationary phase. A $60: 40$ by volume mixture of water and acetonitrile was used as the mobile phase at a flow rate of $1.0 \mathrm{~mL} / \mathrm{min}$. The sample injection volume was $10 \mu \mathrm{L}$. The PTX absorbance was measured using a UV detector at $204 \mathrm{~nm}$ wavelength. Standard solutions containing different concentrations of PTX in the range of $10-1000 \mu \mathrm{g} / \mathrm{mL}$ were prepared from a concentrated stock solution. PTX concentrations were estimated using an isocratic reverse phase HPLC method. From these data, a calibration plot was prepared relating UV absorbance to PTX concentration (SM Figure S3).

\subsection{Drug encapsulation efficiency}

The PTX encapsulation efficiency was defined as: encapsulation efficiency $(\%)=$ (amount initially added - amount remaining in the supernatant after encapsulation) / (amount 
initially added) $\times 100$. The amount of PTX remaining in the supernatant after encapsulation was determined by analyzing the PTX concentration in the supernatant of the centrifuged encapsulation solution by the HPLC method.

\subsection{Gel permeation chromatography (GPC) characterization of PEG-PLA following exposure to X-rays or direct UV-A light}

$0.7 \mathrm{mg}$ of PEG-PLA-coated CWO ("PEG-PLA/CWO”) NPs or $0.7 \mathrm{mg}$ of empty (nonCWO-loaded) PEG-PLA micelles were dispersed in $1.45 \mathrm{~mL}$ of PBS. These samples were irradiated with a single 7 Gy dose of X-rays (XRAD 320, $320 \mathrm{keV}, 2.15 \mathrm{~Gy} / \mathrm{min}$ ) or UV-A lamp light (UVP UVGL-15, $365 \mathrm{~nm}$, exposure time $=11 \mathrm{~s}$, source-to-sample distance $=1 \mathrm{~cm}, \mathrm{UV}$ fluence on sample $=2.8 \mathrm{~J} / \mathrm{cm}^{2}(=4 \mathrm{~W}$ (manufacturer information) $\times 11 \mathrm{~s} /(3.5 \mathrm{~cm} \times 4.5 \mathrm{~cm})$ (illuminated area))). $5 \mathrm{~mL}$ of DCM was added to each of these solutions to extract the PEG-PLA polymer from the aqueous PEG-PLA/CWO suspension. The resulting solutions were vigorously mixed for 10 minutes and centrifuged at 5,000 rpm (equivalent to 3214× g, Eppendorf 5804 (F34-6-38, radius of rotor $=11.5 \mathrm{~cm}$ )) for 20 minutes. The DCM-rich (bottom) phase of the supernatant was collected and dried in a vacuum oven at room temperature for $12 \mathrm{~h}$. The polymer residue was dissolved in HPLC-grade tetrahydrofuran (THF), and the solution was filtered with a $0.22 \mu \mathrm{m}$ PTFE filter. Both X-ray/UV-treated and non-X-ray/UV-treated polymer samples were analyzed using an Agilent Technologies 1200 Series GPC system equipped with a Hewlett-Packard G1362A refractive index (RI) detector and three PLgel $5 \mu \mathrm{m}$ MIXED-C columns. THF was used as the mobile phase at $35.0^{\circ} \mathrm{C}$ and a flow rate of $1 \mathrm{~mL} / \mathrm{min}$. The pristine PEG-PLA (not exposed to X-rays or UV-A light) was used as control.

\subsection{Cell culture}

HN31 cells were generously provided by Dr. Jeffrey N. Myers at MD Anderson Cancer Center [42]. HN31 cells were cultured in Dulbecco's Modified Eagle Medium (DMEM) supplemented with $10 \% \mathrm{v} / \mathrm{v}$ fetal bovine serum and $0.1 \%$ L-glutamine (Gibco Life Technologies) (as recommended by American Type Culture Collection (ATCC)) in a humidified incubator with $5 \% \mathrm{CO}_{2}$ at $37.0^{\circ} \mathrm{C}$. Once the cell confluence reached $80 \%$, the growth medium was removed, and adherent cells were washed twice with PBS (Gibco Life Technologies). Cells were then detached from the plates by treatment with TrypLE ${ }^{\mathrm{TM}}$ Express $(1 \times)$ solution for $4-6$ minutes at $37.0{ }^{\circ} \mathrm{C}$. Detached cells were centrifuged at $300 \times \mathrm{g}$ for 5 minutes at room 
temperature. The cell pellet was resuspended in a minimal amount of growth medium $(2-3 \mathrm{ml})$, and the cells were counted using a haemocytometer. These cells were plated in $\mathrm{T}-25 \mathrm{~cm}^{2}$ flasks (Corning) at a seeding density of $0.2-0.5 \times 10^{6}$ cells per $\mathrm{mL}$ in $5 \mathrm{~mL}$ growth medium.

\subsection{MTT cell viability assay}

The in vitro cytotoxicities of both uncoated CWO NPs (10 nm diameter determined by TEM $[35,36])$ and PEG-PLA-coated CWO NPs (50 nm hydrodynamic diameter determined by DLS) in HN31 cells were evaluated using the MTT assay procedure described in the literature [43]. HN31 cells in the exponential growth phase were seeded in a flat-bottom 96-well polystyrene-coated plate at a seeding density of $0.5 \times 10^{4}$ cells per well, and incubated for 24 hours at $37.0{ }^{\circ} \mathrm{C}$ in a $5 \% \mathrm{CO}_{2}$ incubator prior to exposure to CWO NPs. Cells were then treated with various concentrations of PEG-PLA-coated and uncoated CWO NPs $(0.16,0.32,0.63,1.25$, 2.5 and $5.0 \mathrm{mg}$ CWO per mL solution) $(\mathrm{N}=5)$. After 24 hours of incubation, $10 \mu \mathrm{L}$ of the MTT reagent was added to each well, and further incubated for additional 4 hours. Afterwards, formazan crystals were dissolved by adding $150 \mu \mathrm{L}$ of a $10 \% \mathrm{w} / \mathrm{v}$ SDS solution to each well, and the absorbances at $570 \mathrm{~nm}$ were immediately measured using a microplate reader (BIO-RAD Microplate Reader-550). The wells with no cells, i.e., containing only the DMEM growth medium, the nanoparticles, and the MTT reagent, were used as the blanks. The wells containing cells (that had not been treated with the nanoparticles) in the medium with the MTT reagent were used as controls.

\subsection{Clonogenic cell survival assay}

HN31cells were seeded in 60-mm culture dishes at densities of $0.2 \times 10^{3}$ cells per dish for $0 \mathrm{~Gy}, 1.0 \times 10^{3}$ cells per dish for $3 \mathrm{~Gy}, 2.0 \times 10^{3}$ cells per dish for $6 \mathrm{~Gy}$, and $5.0 \times 10^{3}$ cells per dish for $9 \mathrm{~Gy}$ radiation dose. Samples were prepared in quadruplet for each radiation dose (N =4). Three groups were tested: (1) cells treated for 3 hours with PEG-PLA-coated CWO NPs, (2) cells treated for 3 hours with PTX-loaded PEG-PLA-coated CWO NPs, and (3) untreated cells (control). After 3 hours of nanoparticle treatment, cells were exposed to various doses of $320 \mathrm{keV}$ X-rays at a dose rate of 2.15 Gy per minute (XRAD 320, Precision X-Ray). Irradiated cells were cultured for 14 days (in order to make sure that clonogenically active cells undergo at least 6 generations of cell division). Colonies resulting from radio-resistant cells were stained with Crystal Violet. Colonies of more than 50 daughter cells in culture were counted $(\mathrm{N}=4)$. 
The Plating Efficiency (PE) and the Survival Fraction (SF) were calculated based on the number of such colonies relative to that of the respective non-irradiated subgroup for each group: PE (\%) $=($ number of colonies survived) $/$ (number of cells initially plated $) \times 100 ; \mathrm{SF}(\%)=(\mathrm{PE}$ of a treated group) $/(\mathrm{PE}$ of control $) \times 100$. Survival fraction $(\mathrm{SF})$ vs. radiation dose $(\mathrm{D})$ data were fit to the linear quadratic model, $S F(D)=\exp \left[-\left(\alpha D+\beta D^{2}\right)\right]$, where $\alpha$ and $\beta$ are fit parameters. The Sensitization Enhancement Ratio (SER) was calculated as the ratio of the X-ray dose needed to obtain $10 \%$ survival in untreated cells relative to the dose needed to obtain $10 \%$ survival in nanoparticle-treated cells.

\subsection{HN31 tumor xenografts in NRG mice}

Animal studies were performed in accordance with the guidelines of the American Association for Accreditation of Laboratory Animal Care (AAALAC). Immune-deficient NonObese Diabetic (NOD) Rag Gamma (NRG) mice (6 - 7 weeks old, female) were housed in standard cages within a pathogen-free facility with free access to food and water and an automatic 12-h light-dark cycle. Mice were initially acclimated to the environment for 1 week prior to xenograft implantation. Subcutaneous Head and Neck Squamous Cell Carcinoma (HNSCC) xenografts were produced by implanting $1 \times 10^{6} \mathrm{HN} 31$ cells in $0.1 \mathrm{~mL}$ (total volume) of serum-free medium containing 50\% Matrigel (BD Bioscience) into the mouse flanks (on Day $0)$.

\subsection{Evaluation of antitumor efficacy in mouse HNSCC models}

Three samples (including the candidate formulations and control) were investigated: (i) PEG-PLA/CWO NPs, (ii) PEG-PLA/CWO/PTX NPs (both in sterile PBS solution), and (iii) blank PBS without NPs (negative control). The efficacy of these formulations was assessed following intratumoral (IT) administration in mouse HN31 xenografts (NRG mice, $\mathrm{N}=8$ ) both in the presence and absence of X-ray irradiation. HN31 xenografts were prepared as described above. Once the tumor size reached the $100-150 \mathrm{~mm}^{3}$ level, NP formulations (total $100-150$ $\mu \mathrm{L}$ solution containing $10 \mathrm{mg} / \mathrm{mL}$ of $\mathrm{CaWO}_{4}$ ) were IT administered in two portions over two days (on Day 0 and Day 1) to a final NP concentration of $10 \mathrm{mg} \mathrm{CWO}$ per cc tumor; this NP dose level was adopted based on recent clinical studies by Nanobiotix that tested safety and efficacy of intratumorally delivered $\mathrm{HfO}_{2} \mathrm{NP}$ radio-sensitizers [44]. NP-treated tumors were exposed to total 8 Gy fractionated X-Ray doses (with a daily fraction of 2 Gy repeated over 4 
consecutive days, $\mathrm{t}=1-4$ days). The tumor sizes were measured using a digital caliper at regular intervals. The tumor volume was calculated by the formula, $\mathrm{V}=(\pi / 6) \mathrm{LWH}$ where $\mathrm{L}, \mathrm{W}$ and $\mathrm{H}$ are the length, width and height of the tumor, respectively. Mouse survival analysis was performed using the standard ICH (The International Council for Harmonisation of Technical Requirements for Pharmaceuticals for Human Use) criteria (euthanasia is required if tumor size $>2000 \mathrm{cc}$ or body weight reduction > 20\%). Following euthanization, tumor tissues were collected and wet weighed. Tumor and organ (liver, spleen, lung, heart, kidney, and brain) specimens were also collected for histology analysis.

\subsection{Evaluation of NP biodistribution (BD) in mouse HNSCC models}

The BD of CWO NPs were investigated in HN31 xenograft-bearing NRG mice (3 mice per treatment group; see Section 2.9 above) following IT administration of PEG-PLA/CWO/PTX NPs $(\mathrm{N}=3)$. The time-dependent CWO concentrations in tumor, blood and other selected tissues were measured by atomic absorption spectroscopy (AAS) as previously described [36]. The following specific procedures were used. Total 21 mice were divided into 7 groups (Groups I VII) with 3 mice per group. Mice in Groups I - VI received IT injections of PEGPLA/CWO/PTX NPs at Days 0 and 1 (as described in Section 2.10), whereas mice in Group VII received only PBS via IT route at Days 0 and 1 (control). NP/PBS-injected mice were treated with 2 Gy daily fractions of $320 \mathrm{keV}$ X-rays during first 4 days (i.e., at Days 1, 2, 3 and 4, up to total 8 Gy X-ray dose). Groups I, II, III, IV, V and VI was sacrificed by euthanization at Day 1, 3, 5, 7, 14 and 30, respectively. The cumulative X-Ray doses mice received were 2 Gy for Group I, 4 Gy for Group II, and 8 Gy for all other Groups (III - VI). Control mice (Group VII) were euthanized at Day 1. Tumor and organ (liver, spleen, kidneys, lungs, brain, and heart) were collected after euthanization. Tissue samples were processed for AAS analysis using the published procedure[36].

\subsection{Statistical analysis}

All in vitro measurements were performed in minimum triplicates. Different animal numbers were chosen for different in vivo assays based on our experience and needs in terms of statistical significance. All data are presented as mean \pm standard deviation. A one-way ANOVA was used to determine whether there was a statistically significant difference in effect between 
different treatment groups for data presented in Figures 4, 5 and 6. Kaplan-Meier survival analysis was used to plot unadjusted survival of mice treated with different formulations; results (Figure 7) were analyzed using the log-rank test. Difference was considered statistically significant if $\mathrm{p}<0.05(*)$ and highly significant if $\mathrm{p}<0.01(* *)$.

\section{RESULTS \& DISCUSSION}

\subsection{Paclitaxel release kinetics}

As explained in Scheme 1, CWO NPs are coated with PEG-PLA polymers. Hydrophobic PLA chains form a globular domain wherein CWO NPs are encapsulated. Hydrophilic PEG chains form a hydrated brush layer. Water-insoluble PTX molecules are co-encapsulated within the hydrophobic PLA domain. The amount of PTX released from PEG-PLA-coated CWO NPs was measured by HPLC for 32 days; both X-ray-irradiated and non-irradiated samples were tested. As control, PTX release from PEG-PLA micelles (containing no co-encapsulated CWO NPs) was also quantitated. Notably, it was discovered that in the absence of radiation, PEGPLA/CWO/PTX NPs showed the lowest PTX release; about 75\% PTX remained unreleased at Day 32 (Figure 2(A)). In contrast, upon exposure to 7 Gy X-Ray dose, a sudden rapid release of PTX was observed (that is, close to $70 \%$ of the initially loaded PTX amount was released within 3 days following $\mathrm{X}$-ray irradiation); this radiation-triggered rapid release phase was followed by a slower release phase over the remaining non-irradiated period (Figure 2(A)). At the end of the 30-day period following radiation, a total of about $90 \%$ of the loaded PTX was released. In contrast, in the PTX-loaded PEG-PLA micelle case (involving no co-encapsulated CWO NPs), the PTX release profile was significantly less affected by X-ray irradiation (Figure 2(B)). These results indicate that the process of radiation-triggered PTX release from PEG-PLA/CWO/PTX NPs is enabled by the co-encapsulation of CWO NPs. Figure S4 displays a comparison of PTX release profiles between non-irradiated PTX-loaded PEG-PLA micelles in the presence and absence of co-encapsulated CWO NPs (redrawn from Figures 2(A) and 2(B)). These data suggest that when co-encapsulated with CWO NPs, PTX molecules are encapsulated into the deeper region of the PLA layer. However, PTX is rapidly released upon X-ray irradiation (Figure 2(A)) because of PLA degradation under X-ray irradiation (Section 3.2). 
The PTX release rate was found to be proportional to radiation dose. As shown in Figure 2(A), when a lower X-Ray dose (2 Gy) was used, the slope of the PTX release profile in the rapid release phase was proportionally decreased; in the 2 Gy case, a little over $30 \%$ of the initially loaded PTX amount was released within 3 days following X-ray irradiation. At the end of the 30-day period post radiation, a total of close to $40 \%$ of the loaded PTX was released.
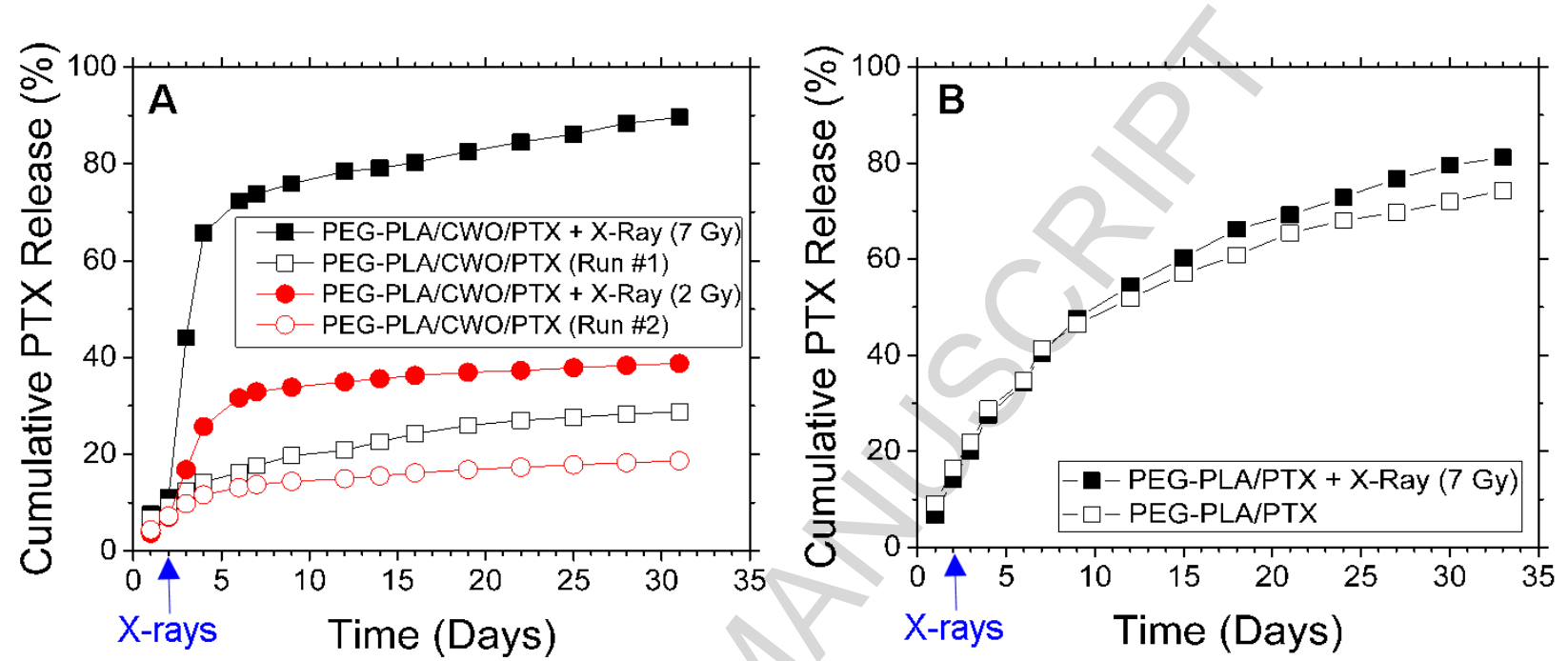

Figure 2. (A) Cumulative PTX release profiles for X-ray-irradiated vs. non-X-ray-irradiated PEG-PLA/CWO/PTX NPs over 32 days. NPs were irradiated with a single 7 or 2 Gy dose of $320 \mathrm{keV}$ X-rays at Day 2 . Over $50 \%$ PTX release occurred during Days $2-4$ following X-ray irradiation. (B) Cumulative PTX release profiles for X-rayirradiated vs. non-X-ray-irradiated PEG-PLA micelles loaded with PTX (with no co-encapsulated CWO NPs) over 33 days. The PTX-loaded PEG-PLA micelles were irradiated with a single 7 Gy dose of $320 \mathrm{keV}$ X-rays at Day 2. X-ray irradiation only had a small influence on PTX release from PEG-PLA micelles.

\subsection{Photo-lytic degradation of PLA}

Under X-ray irradiation, UV-A is generated by CWO NPs. Radiation causes the release of PTX from the PLA coating layer into the aqueous surrounding (Figure 2(A)). We believe that this PTX release triggered by X-rays is due to the degradation of the PLA polymer that occurs under X-ray irradiation. In order to confirm the degradation of PLA under X-ray irradiation, GPC measurement was performed on the PEG-PLA re-extracted with chloroform from PEGPLA-coated CWO NPs following exposure to X-rays (320 keV, 7 Gy) ("PEG-PLA/CWO + XRay") (Figure 3). In Figure 3, a GPC trace for pristine PEG-PLA ("PEG-PLA") is also displayed for comparison. Also of note, no difference in GPC curves was observed between pristine PEGPLA and the PEG-PLA re-extracted from non-X-ray-exposed PEG-PLA-coated CWO NPs (data not shown). As shown in the figure, the X-ray-exposed sample ("PEG-PLA/CWO + X-ray") 
showed an overall shift of the peak to, and a shoulder developed, at longer elution times (on the lower molecular weight side), which clearly indicates that the degradation of the polymer occurred; the PTX release triggered by X-ray radiation is due to the chemical degradation of the encapsulating polymer (not due to physical excitation processes).

It should be noted that data shown in Figure 2(B) suggest that X-rays alone may also cause slight chemical change in PLA. It has been reported in the literature that short wavelength UV light (100 - $280 \mathrm{~nm}$ UV-C, and $280-320 \mathrm{~nm} \mathrm{UV-B})$ can directly cause degradation of PLA [45-48]. However, it is a reasonable question whether the secondary UV-A light generated by CWO NPs under X-ray irradiation by itself (having a peak wavelength of about $400 \mathrm{~nm}$ ) can effectively cause polymer degradation, particularly at the low UV-A dose level produced by 7 Gy of $320 \mathrm{keV} \mathrm{X-ray} \mathrm{radiation}\left(\approx 0.561 \mathrm{~J} / \mathrm{cm}^{2}\right)$ [49]. Yet another possible mechanism is that CWO plays a catalytic role in the X-ray-induced PLA degradation process. It has been known that CWO has photo-catalytic activity under UV-A light; under the influence of UV-A light, CWO catalyzes the degradation of water and oxygen molecules in the surrounding environment [50]. However, CWO-mediated photo-catalytic degradation of PLA has not previously been demonstrated. To better understand the exact mechanism of the PLA degradation in X-rayirradiated PEG-PLA/CWO NPs, additional GPC measurements were made on (i) PEG-PLA reextracted from PEG-PLA-coated CWO NPs following exposure to direct UV-A light (365 nm, $2.8 \mathrm{~J} / \mathrm{cm}^{2}$, equivalent $365 \mathrm{~nm}$ UV-A fluence generated by PEG-PLA/CWO NPs under 7 Gy 320 keV X-ray radiation) ("PEG-PLA/CWO + UV-A”), (ii) PEG-PLA re-extracted from empty (nonCWO-loaded) PEG-PLA micelles following exposure to X-rays (320 keV, 7 Gy) ("PEG-PLA + X-Ray"), and (iii) PEG-PLA re-extracted from empty (non-CWO-loaded) PEG-PLA micelles following exposure to direct UV-A light (365 nm, $2.8 \mathrm{~J} / \mathrm{cm}^{2}$ ) ("PEG-PLA + UV-A"). The results are also presented in Figure 3. As shown in the figure, both X-rays alone ("PEG-PLA + X-Ray") and UV-A light alone ("PEG-PLA + UV-A") caused PLA degradation even in the absence of CWO NPs. Further, the extents of PLA degradation were comparable between "PEG-PLA + UV-A" and "PEG-PLA/CWO + UV-A", and also between "PEG-PLA + X-Ray" and "PEGPLA/CWO + X-Ray". These results indicate that CWO does not produce any significant catalytic activity for PLA degradation; the PLA degradation is therefore not of photo-catalytic type, but it is a photo-lysis reaction. We speculate that this PLA degradation reaction is essentially a hydrolysis process. Although insoluble in water, PLA is known to absorb water up 
to about $1 \%$ by weight [51]. UV-A/X-ray radiation generates reactive oxygen species (such as hydroxyl radicals) via UV-A/radiolysis of water $[52,53]$, which reacts with and cleaves the ester bonds in PLA.

Based on the results presented in this and previous sections, we speculate more specifically that the mechanism of radiation-induced PTX release involves two processes: (i) partitioning/accumulation of PTX in the vicinity of the $\mathrm{CaWO}_{4}$ core (causing very slow release of PTX in the absence of radiation) (Figure 2(A)), and (ii) photo-lytic degradation of PLA (triggering rapid PTX release under X-ray irradiation) (Figure 3). Regarding (i), we believe that the poor water solubility of PTX $(\approx 0.1-0.3 \mu \mathrm{g} / \mathrm{ml})[54]$ is the key parameter contributing to the accumulation of PTX in the deeper region of the PLA domain; because of its poor water solubility, PTX precipitates to the $\mathrm{CaWO}_{4}$ surface earlier than did PLA during the solvent exchange process. Therefore, similar chemotherapeutic formulations are possible with other types of chemo drugs, as long as the drug is similarly poorly water-soluble. Further study is needed to test this possibility. 


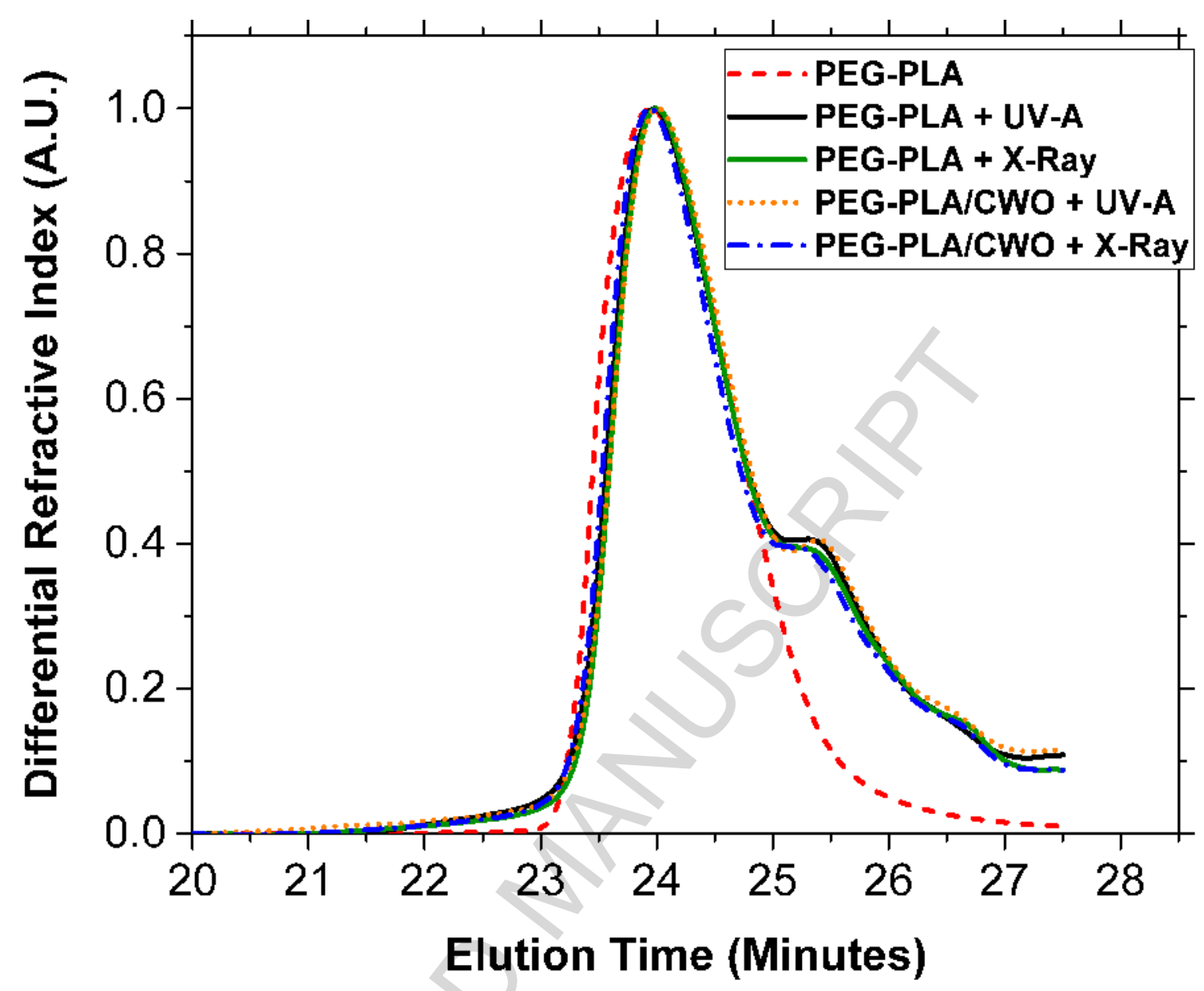

Figure 3. GPC traces of (1) pristine PEG-PLA ("PEG-PLA"), (2) PEG-PLA re-extracted from PEG-PLA-coated CWO NPs following exposure to X-rays (320 keV, 7 Gy) ("PEG-PLA/CWO + X-Ray"), (3) PEG-PLA re-extracted from PEG-PLA-coated CWO NPs following exposure to direct UV-A light $\left(365 \mathrm{~nm}, 2.8 \mathrm{~J} / \mathrm{cm}^{2}\right.$ (of note this UV-A fluence used is significantly higher than the equivalent $365 \mathrm{~nm}$ UV-A fluence generated by PEG-PLA/CWO NPs under 7 Gy $320 \mathrm{keV}$ X-ray radiation $\left(\approx 0.561 \mathrm{~J} / \mathrm{cm}^{2}\right.$ [49]))) ("PEG-PLA/CWO + UV-A"), (4) PEG-PLA re-extracted from empty (non-CWO-loaded) PEG-PLA micelles following exposure to X-rays (320 keV, 7 Gy) ("PEG-PLA + XRay"), and (5) PEG-PLA re-extracted from empty (non-CWO-loaded) PEG-PLA micelles following exposure to direct UV-A light (365 nm, $\left.2.8 \mathrm{~J} / \mathrm{cm}^{2}\right)$ ("PEG-PLA + UV-A").

\subsection{Non-cytotoxicity of PEG-PLA CWO NPs}

In vitro cytotoxicities of uncoated CWO NPs (10 nm diameter) and PEG-PLAencapsulated CWO NPs (50 nm hydrodynamic diameter) were evaluated in HN31 (p53-mutant human head and neck cancer) cells using the standard MTT protocol at various CWO concentrations ranging from 0.16 to $5 \mathrm{mg} / \mathrm{ml}$. The results are presented in Figure 4. As shown in the figure, no significant toxicity was observed for both samples up to a nominal CWO concentration of about $1.25 \mathrm{mg} / \mathrm{ml}$. At higher concentrations $(2.5$ and $5 \mathrm{mg} / \mathrm{ml})$, a slight 
reduction $(10-20 \%)$ in viability was observed. It should be noted that the actual CWO concentration that the cells experience is typically significantly higher than the nominal value of CWO concentration because of the sedimentation of the CWO NPs that occurred during the 24-h incubation period. The nominal NP concentration value of, for instance, $1.25 \mathrm{mg}$ of CWO per ml of solution used in the cell culture study is equivalent to total $50 \mathrm{ng}$ of CWO per HN31 cell (0.20 $\mathrm{ml}$ of medium used per well having a surface area of $32 \mathrm{~mm}^{2}$ and a cell confluence of 156 cells $/ \mathrm{mm}^{2}$ ). The time it takes for a PEG-PLA/CWO/PTX NP (of $66 \mathrm{~nm}$ hydrodynamic diameter) to sediment a distance of $0.625 \mathrm{~cm}$ (the height of the cell culture medium in our study) is estimated to be about 51.8 days, using the equation, $\tau \approx 91 \eta /\left(2 R^{2} \Delta \rho g\right)$, where $\tau$ is the sedimentation time, 1 is the sedimentation distance, $\eta$ is the viscosity of the medium (water), $R$ is the radius of the NP, $\Delta \rho$ is the density difference between the NP and medium, and $g$ is the gravitational acceleration constant. Therefore, we expect that by the end of the 24-hour incubation period, about $1.9 \%$ of the NPs in the system have sedimented to the bottom of the well, which gives an estimate of $0.97 \mathrm{ng}$ of CWO per HN31 cell for the actual CWO concentration that the cells experience at the bottom of the plate. As explained in Section 2.10, the NP dose level used in our in vivo study (results to be discussed in Section 3.5) was $10 \mathrm{mg}$ of CWO compound per cc of tumor. Assuming an HN31 cell density of $10^{9}$ cells per cc of tumor for mouse HN31 xenograft tissue [55], the NP concentration used in our in vivo study (10 mg of CWO per cc of tumor) is converted to a value of $0.010 \mathrm{ng}$ of CWO per HN31 cell, which is about two orders of magnitude lower than the cytotoxicity threshold estimated by the in vitro method (0.97 ng of CWO per cell).

Therefore, the results presented in Figure 4 clearly support that CWO NPs, regardless of whether PEG-PLA-coated or uncoated, are completely non-cytotoxic at therapeutically relevant NP doses. 

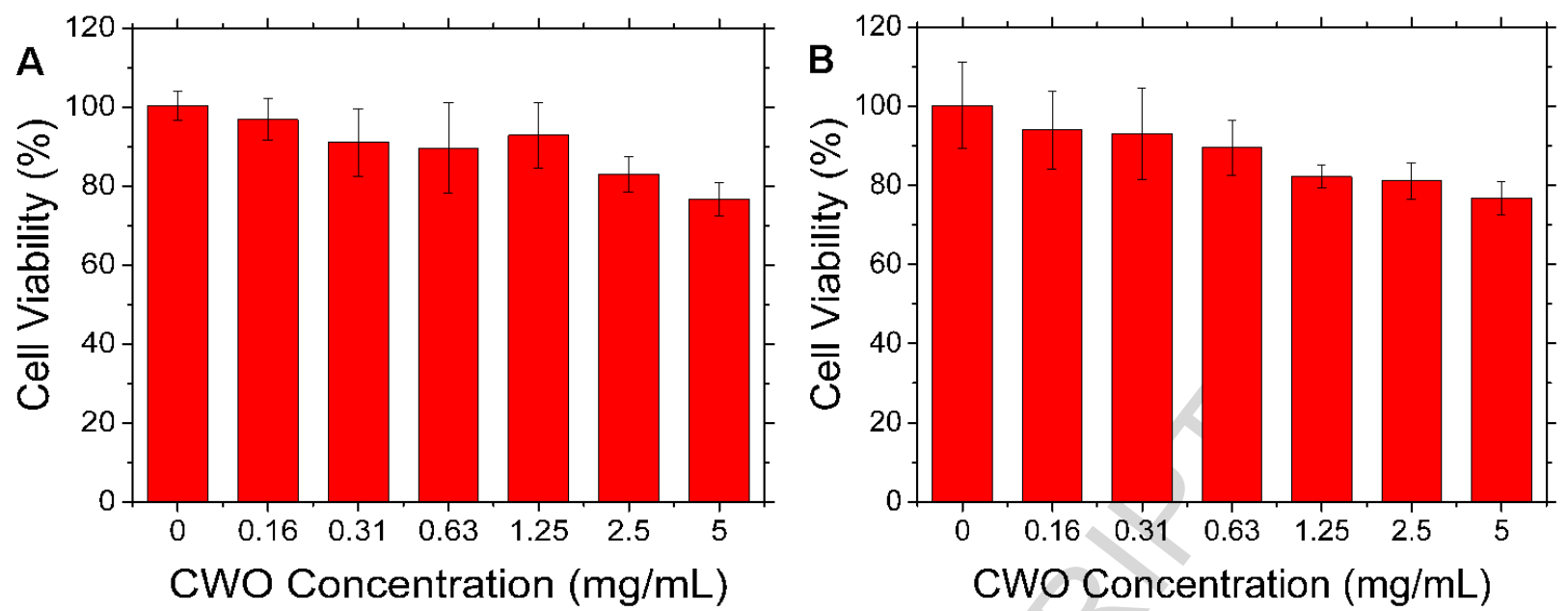

Figure 4. In vitro cytotoxicities of (A) uncoated CWO NPs (10 nm diameter) and (B) PEG-PLA-coated CWO NPs (50 $\mathrm{nm}$ hydrodynamic diameter) in HN31 cells assessed by the MTT method ( $\mathrm{N}=5)$. HN31 cells were seeded on 96-well culture plates at a density of $0.5 \times 10^{4}$ cells per well and incubated for 24 hours prior to exposure to nanoparticles. The MTT measurements were performed at $24 \mathrm{~h}$ post nanoparticle treatment. The p-values for all pairs of groups are summarized in Table 1 below.

Table 1. Results of the statistical analysis of - that is, p-values for - the tumor growth data presented in Figures 4(A) (upper table) and 4(B) (lower table) $(\mathrm{N}=5)$.

\begin{tabular}{|c|l|l|l|l|l|l|}
\hline CWO Concentration $(\mathrm{mg} / \mathrm{mL})$ & 0.16 & 0.31 & 0.63 & 1.25 & 2.5 & 5.0 \\
\hline 0 & 0.384 & 0.157 & 0.194 & 0.223 & 0.006 & 0.002 \\
\hline 0.16 & - & 0.378 & 0.378 & 0.523 & 0.025 & 0.006 \\
\hline 0.31 & - & - & 0.867 & 0.813 & 0.22 & 0.058 \\
\hline 0.63 & - & - & - & 0.714 & 0.402 & 0.139 \\
\hline 1.25 & - & - & - & - & 0.147 & 0.041 \\
\hline 2.5 & - & - & - & - & - & 0.151 \\
\hline
\end{tabular}

\begin{tabular}{|c|l|l|l|l|l|l|}
\hline CWO Concentration (mg/mL) & 0.16 & 0.31 & 0.63 & 1.25 & 2.5 & 5.0 \\
\hline 0 & 0.505 & 0.478 & 0.227 & 0.051 & 0.045 & 0.029 \\
\hline 0.16 & - & 0.918 & 0.558 & 0.12 & 0.11 & 0.049 \\
\hline 0.31 & - & - & 0.677 & 0.191 & 0.173 & 0.083 \\
\hline 0.63 & - & - & - & 0.171 & 0.157 & 0.053 \\
\hline 1.25 & - & - & - & - & 0.742 & 0.137 \\
\hline 2.5 & - & - & - & - & - & 0.292 \\
\hline
\end{tabular}

\subsection{Clonogenic survival following various doses of radiation in HN31 cells treated with concurrent PEG-PLA/CWO/PTX NPs}

An in vitro clonogenic study was performed to determine whether PEG-PLA/CWO/PTX

NPs are indeed capable of inducing a significant enhancement of the tumor suppressive effect of $\mathrm{X}$-rays $/ \gamma$ rays beyond what is achievable with PEG-PLA/CWO NPs (i.e., without co-delivered PTX). Again, the HN31 cell line was used for this investigation. As shown in Figure 5, 
concomitant PEG-PLA/CWO/PTX NPs produced a significantly greater radiation sensitization effect than concomitant PEG-PLA/CWO NPs. The values of the Sensitizer Enhancement Ratio or SER (defined as the ratio of the radiation dose at $10 \%$ clonogenic survival in the absence of nanoparticles relative to the radiation dose at $10 \%$ survival in the presence of nanoparticles) were estimated to be 1.16 for concomitant PEG-PLA/CWO NPs (i.e., without PTX) and 1.45 for PEGPLA/CWO/PTX NPs (with co-encapsulated PTX).

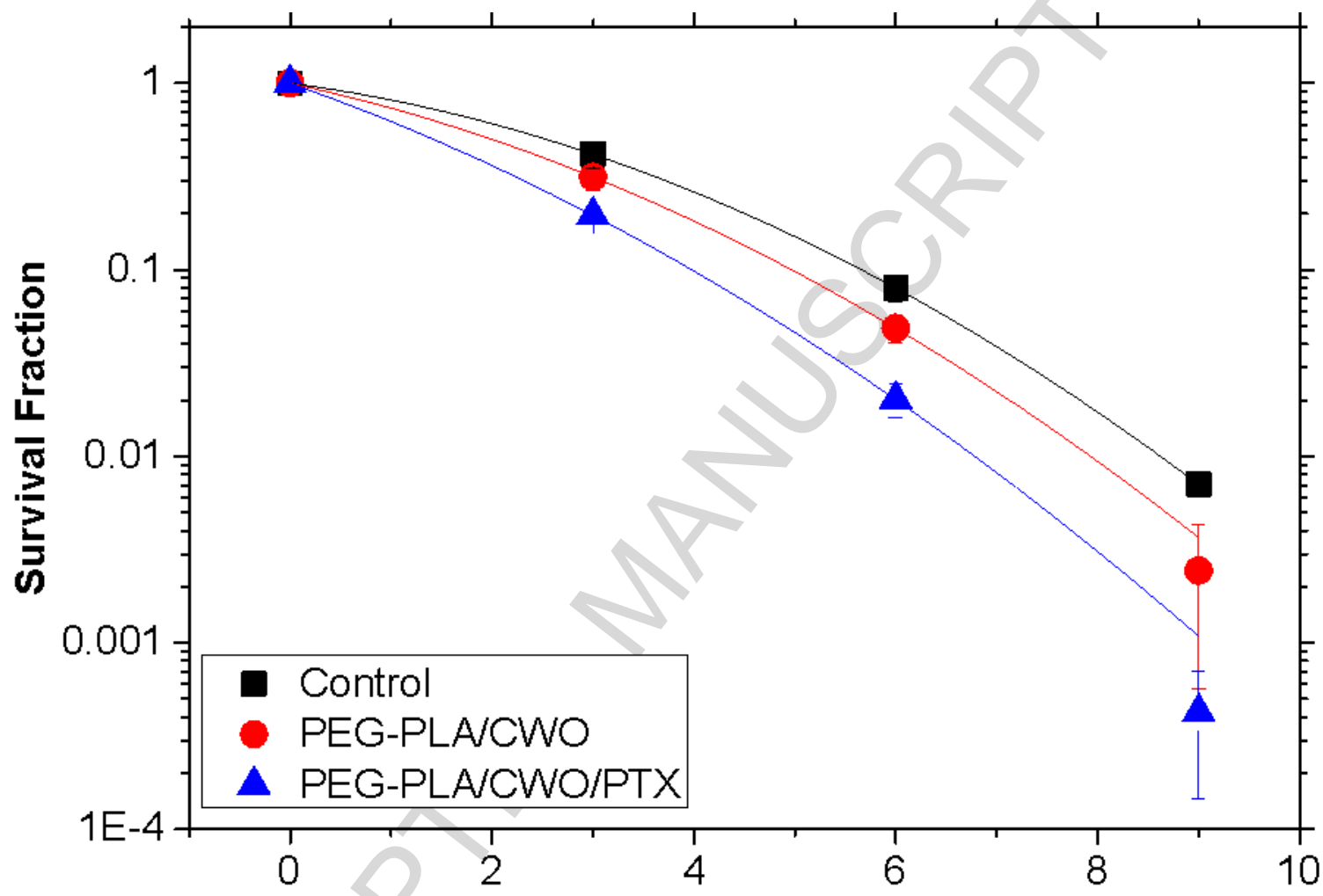

\section{Radiation Dose (Gy)}

Figure 5. Effect of X-ray irradiation on clonogenic survival of HN31 cells at various X-ray doses. HN31 cells were irradiated in the presence of PEG-PLA/CWO/PTX NPs $($ CWO concentration $=0.20 \mathrm{mg} / \mathrm{ml}$, PTX concentration $=$ $0.023 \mathrm{mg} / \mathrm{ml}$, PEG-PLA:CWO:PTX $=1.3: 1.0: 0.12$ by weight (determined by AAS, HPLC and TGA)) or PEGPLA/CWO NPs (CWO concentration $=0.20 \mathrm{mg} / \mathrm{ml}$, PEG-PLA:CWO $=1.3: 1.0$ by weight (determined by AAS and TGA [35])). HN31 cells were seeded on $60 \mathrm{~mm}$ culture plates at densities $0.2 \times 10^{3}(0 \mathrm{~Gy}), 1.0 \times 10^{3}$ (3 Gy), $2.0 \times$ $10^{3}(6 \mathrm{~Gy})$ and $5.0 \times 10^{3}(9 \mathrm{~Gy})$ cells per plate. After $24 \mathrm{~h}$ incubation with nanoparticles, cells were exposed to various doses of $320 \mathrm{keV}$ X-ray radiation. All doses were given in single fractions. Irradiated cells were cultured for 14 days. Colonies resulting from radio-resistant cells were stained by Crystal Violet. Colonies of more than 50 daughter cells in culture were counted $(\mathrm{N}=4)$. Table 2 below summarizes the linear quadratic fit results and the SER (Sensitization Enhancement Ratio estimated at $10 \%$ clonogenic survival) values. The p-values for all pairs of groups are summarized in Table 3.

Table 2. Summary of the results of the analysis of the data shown in Figure 4. $\alpha$ and $\beta$ are the linear-quadratic exponential fit parameters. The Sensitization Enhancement Ratio or SER is defined as the ratio of the radiation dose at $10 \%$ clonogenic survival in the absence of $\mathrm{CWO}$ relative to the radiation dose at $10 \%$ survival in the presence of CWO.

\begin{tabular}{|l|l|l|c|}
\hline$\alpha$ & $\beta$ & $\alpha / \beta$ & SER \\
\hline
\end{tabular}




\begin{tabular}{|l|l|l|l|l|}
\hline Control & 0.159 & 0.044 & 3.61 & 1 \\
\hline PEG-PLA/ CWO NP & 0.266 & & & 1.15 \\
\hline $\begin{array}{l}\text { PEG-PLA-PTX/CWO } \\
\text { NP }\end{array}$ & 0.436 & 0.039 & 6.82 & \\
\hline
\end{tabular}

Table 3. Results of the statistical analysis of - that is, p-values for - the tumor growth data presented in Figure 5 (N $=4)$.

\begin{tabular}{|l|l|l|l|}
\hline Radiation Dose (Gy) & Formulation & PEG-PLA/CWO NP & PEG-PLA/CWO/PTX NP \\
\hline \multirow{3}{*}{3} & Control & 0.191 & 0.006 \\
\cline { 2 - 4 } & PEG-PLA/CWO NP & - & 0.0012 \\
\hline \multirow{3}{*}{9} & Control & 0.02 & 0.0012 \\
\cline { 2 - 4 } & PEG-PLA/CWO NP & - & 0.0052 \\
\cline { 2 - 4 } & Control & 0.716 & 0.599 \\
\cline { 2 - 4 } & PEG-PLA/CWO NP & - & 0.44 \\
\hline
\end{tabular}

The clonogenic survival curves for radiated HN31 cells (regardless of whether X-rays were used alone or in combination with concomitant PEG-PLA/CWO/PTX or PEG-PLA/CWO NPs) were seen to follow the standard exponential-quadratic decay formula:

$$
\mathrm{SF}(\mathrm{D})=\exp \left[-\left(\alpha \mathrm{D}+\beta \mathrm{D}^{2}\right)\right]
$$

where SF is the survival fraction, $\mathrm{D}$ is the $\mathrm{X}$-ray dose, and $\alpha$ and $\beta$ are adjustable parameters for fitting data to the model. Best fit curves are shown as solid curves in Figure 5, and fitting results are summarized in Table 2. It is notable in Figure 5 and Table 2 that even in the absence of coencapsulated PTX, PEG-PLA/CWO NPs were able to induce a significant enhancement of the tumor-suppressive effect of $\mathrm{X}$-rays $(\mathrm{SER} \approx 1.15$ ); this result is consistent with previous data obtained with PEG-PLA/CWO NPs with slightly larger diameter (170 nm hydrodynamic diameter) under identical radiation conditions (SER $\approx 1.10)$ [40]. The incorporation of PTX in the NP formulation further significantly increased the effect of radiation (SER $\approx 1.40$, Figure 5 and Table 2). The $\alpha / \beta$ ratio also has a useful meaning; this ratio represents a radiation dose at which the exponential-linear cell kill effect becomes equivalent in magnitude to the exponentialquadratic cell kill effect of radiation (at $D<\alpha / \beta$ the exponential-linear effect is dominant, whereas at $\mathrm{D}>\alpha / \beta$ the exponential-quadratic effect takes over (the surviving fraction drops more rapidly)). It is generally known that [56]:

- Cells that respond to radiation early have high $\alpha / \beta$ ratios; cell kill linearly increases at low radiation doses. The average value of $\alpha / \beta$ for early responding cells is about 10 ; 
- Cells that respond late have low $\alpha / \beta$ ratios; cell kill is less at low doses, and it greatly increases at high doses. The average value of $\alpha / \beta$ for late responding cells is about 3 ;

- Most tumor cells have high $\alpha / \beta$ ratios (equal to or greater than 10 ). However, some tumor types exhibit much lower ratios; for instance, prostate and melanoma/sarcoma typically show $\alpha / \beta$ values around 3 and 1 , respectively. Tumors with low $\alpha / \beta$ ratios are resistant to low doses of radiation.

Therefore, if chemo drug addition to radiotherapy increases the SER, while it does not affect the $\alpha / \beta$ ratio, chemotherapy can be said to have simply produced an additive therapeutic effect. However, if both the SER and the $\alpha / \beta$ ratio are increased, the cells have become more sensitive to low doses of radiation [56]. As shown in Table 2, concomitant PEG-PLA/CWO/PTX NPs significantly increased the value of the $\alpha / \beta$ ratio $(\alpha / \beta \approx 12.11)$ relative to non-nanoparticle-treated control $(\alpha / \beta \approx 3.61)$, which suggests that the PEG-PLA/CWO/PTX treatment enhanced the radio responsiveness of HN31 cells at low X-ray doses. Therefore, it can be deduced that PTX released from nanoparticles under $\mathrm{X}$-ray irradiation contributed to overall cell kill by increasing radiotherapy efficacy (i.e., by radio sensitization) in addition to functioning as chemotherapy. It should also be noted that, though lesser in degree than PEG-PLA/CWO/PTX NPs, non-PTXloaded PEG-PLA/CWO NPs also increased both SER and $\alpha / \beta$, which supports that PEGPLA/CWO NPs themselves are also an effective radio-sensitizer [40]. This means, by using PEGPLA/CWO/PTX NPs, an improved therapeutic effect can be achieved with the same amount of radiation or the same therapeutic effect can be achieved with significantly less radiation.

\subsection{Therapeutic efficacy of PEG-PLA/CWO/PTX NPs in mouse HN31 xenografts: Tumor growth, and mouse survival}

The therapeutic efficacy of intratumorally administered PEG-PLA/CWO/PTX NPs was evaluated in HN31 mouse xenografts in vivo. For these studies, mice were treated via intratumoral injection with either PTX-loaded ("PEG-PLA/CWO/PTX") NPs, non-PTX-loaded ("PEG-PLA/CWO") NPs or NP vehicle (PBS). Each treatment/control group was divided into two subgroups; one subgroup was treated with a sub-therapeutic dose of $\mathrm{X}$-rays $(320 \mathrm{keV}$, total 8 Gy, 4 fractions of 2 Gy given one fraction per day), and the other was not given X-rays. Tumor growth and mouse survival were measured over time. The results are summarized in Figures 6 (tumor growth) and 7 (mouse survival). 


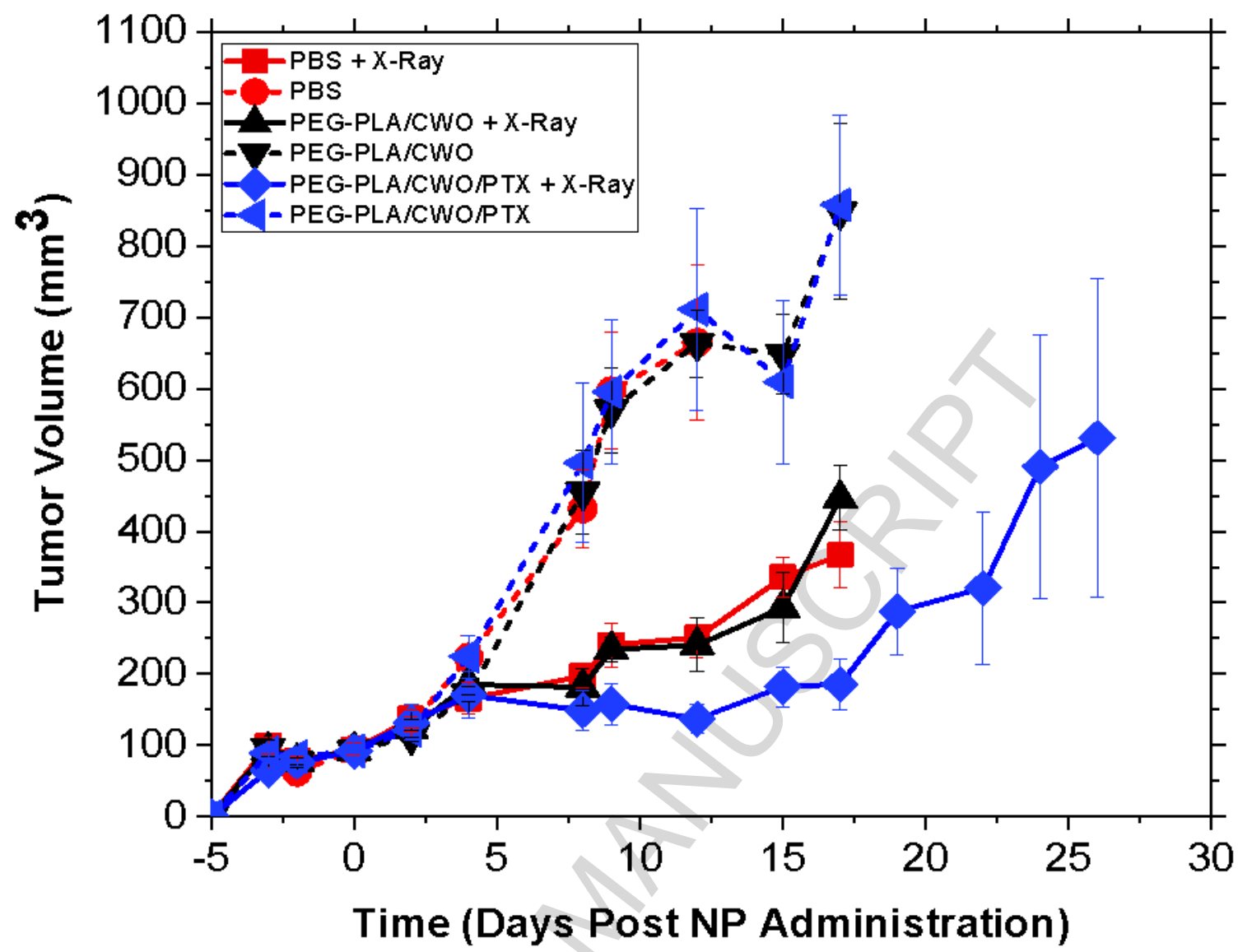

Figure 6. Tumor growth in mice treated with concomitant radiation plus PEG-PLA/CWO/PTX NPs. NRG mice (68 weeks old, female, $\mathrm{N}=8$ per group) were implanted with HN31 cells at Day -5 . Tumors were grown to 0.10 to $0.15 \mathrm{cc}$ until Day 0. Nanoparticles were intratumorally administered in 2 portions at Days 0 and 1 (total $10 \mathrm{mg}$ of CWO per cc of tumor, and total $1.2 \mathrm{mg}$ of PTX per cc of tumor; PEG-PLA:CWO:PTX = 1.3:1.0:0.12 by weight (determined by AAS, HPLC and TGA)). Tumors were irradiated with $320 \mathrm{keV} \mathrm{X-rays} \mathrm{(total} \mathrm{dose} 8 \mathrm{~Gy}$ ) in $2 \mathrm{~Gy} / \mathrm{day}$ fractions over 4 days (at Days 1,2, 3 and 4). Control group was treated with sterile PBS. For all treatment types (PEG-PLA/CWO/PTX, PEG-PLA/CWO, and Control), non-X-ray-treated animals were also included in the study for comparison. For each group tumor size data are shown up to the time point at which any one mouse died or was sacrificed because of tumor overgrowth $\left(>2000 \mathrm{~mm}^{3}\right.$ ) or excessive mouse weight loss $(>20 \%)$. The same criteria were used for the survival analysis shown in Figure 7. Error bars represent standard deviations. The p-values for all pairs of groups are summarized in Table 4 below.

Table 4. Results of the statistical analysis of - that is, p-values for-the tumor growth data presented in Figure 6 (N $=8)$.

\begin{tabular}{|c|c|c|c|c|c|c|}
\hline & PBS & $\begin{array}{l}\text { PEG- } \\
\text { PLA/CWO }\end{array}$ & $\begin{array}{l}\text { PEG- } \\
\text { PLA/CWO/PTX }\end{array}$ & $\begin{array}{l}\text { PBS + } \\
\text { X-Ray }\end{array}$ & $\begin{array}{l}\text { PEG- } \\
\text { PLA/CWO + } \\
\text { X-Ray }\end{array}$ & $\begin{array}{l}\text { PEG- } \\
\text { PLA/CWO/PTX + } \\
\text { X-Ray }\end{array}$ \\
\hline PBS & - & 0.023 & 0.022 & 0.007 & 0.007 & 0.005 \\
\hline PEG-PLA/CWO & - & - & 0.023 & 0.008 & 0.008 & 0.006 \\
\hline $\begin{array}{l}\text { PEG- } \\
\text { PLA/CWO/PTX }\end{array}$ & - & - & - & 0.007 & 0.007 & 0.005 \\
\hline PBS + X-Ray & - & - & - & - & 0.0002 & 0.0001 \\
\hline
\end{tabular}




\begin{tabular}{l|l|l|l|l|l|l|} 
PEG-PLA/CWO + & & & - & - & & 0.0001 \\
X-Ray & - & - & - & 0.0 \\
\hline
\end{tabular}

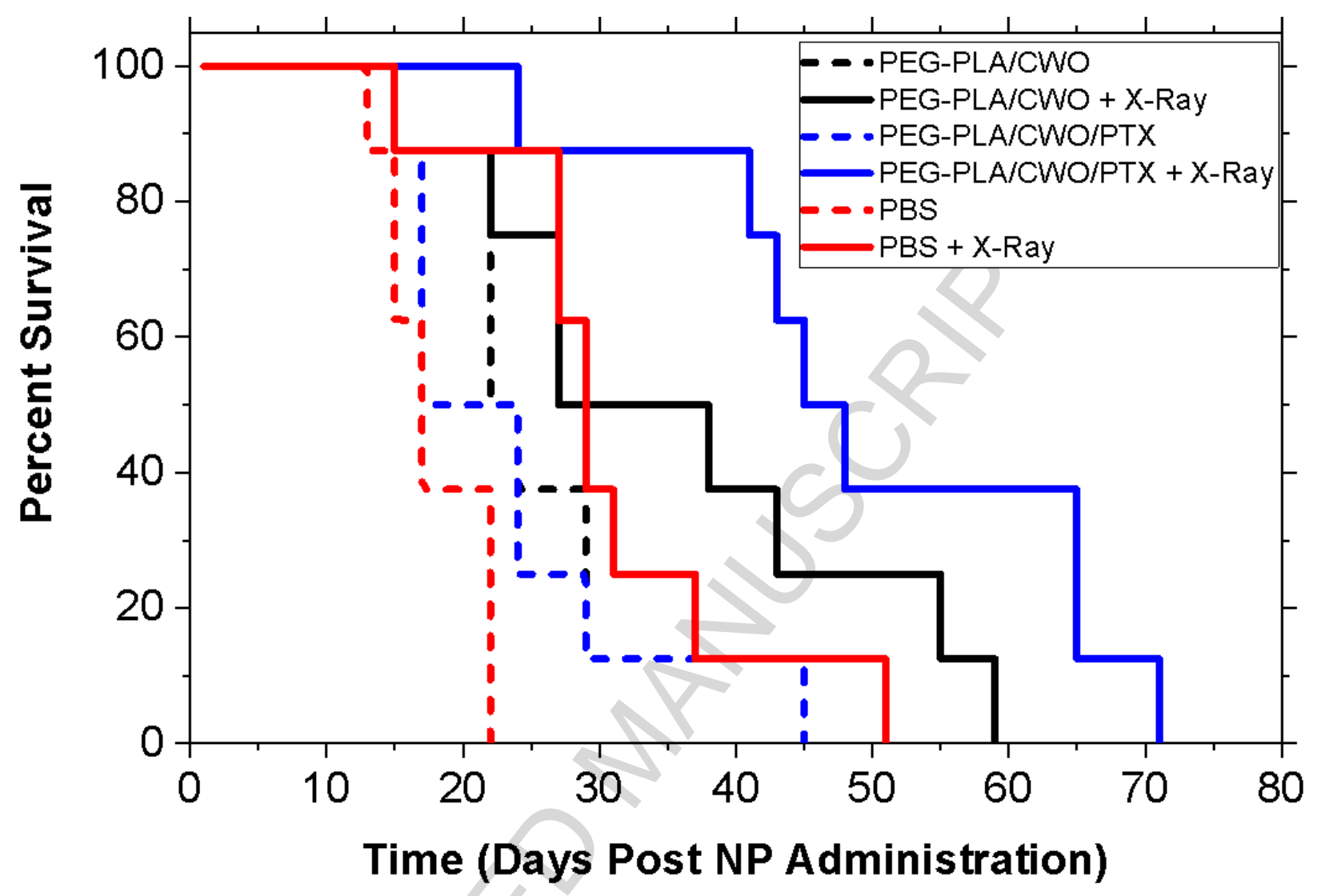

Figure 7. Kaplan-Meier curves for survival of mice $(\mathrm{N}=8)$ treated with PEG-PLA/CWO/PTX NPs, PEGPLA/CWO NPs, and PBS (control) with and without X-rays. PBS solutions of NPs were injected into HN31 xenografts $(0.10-0.15 \mathrm{cc})$ in NRG mice to a final NP concentration of $10 \mathrm{mg}$ of CWO per cc of tumor and a final PTX concentration of $1.2 \mathrm{mg}$ of PTX per cc of tumor (PEG-PLA:CWO:PTX = 1.3:1.0:0.12 by weight (determined by AAS, HPLC and TGA)). A total radiation dose of $8 \mathrm{~Gy}$ was given in 4 fractions of 2 Gy per fraction, one fraction per day over 4 days (at $t=1,2,3$ and 4 days) following NP administration (at $t=0$ and 1 day). Mice were euthanized based on the standard ICH criteria: (a) tumor volume $>2.0 \mathrm{cc}$; (b) body weight loss $>20 \%$ of the original body weight. Analysis of survival data was performed using the log-rank test. Values of $p<0.05$ were considered statistically significant. The PEG-PLA/CWO/PTX + X-Ray group (blue solid line) and PEG-PLA/CWO + X-Ray group (black solid line) were significantly different from the Control (PBS with no X-Ray) group and also from the NPs with no X-Ray groups ( $<0.05$ for each pair-wise comparison). The p-values for all pairs of groups are summarized in Table 5. The median survival times were: 18 days for "PBS", 22 days for "PEG-PLA/CWO", 22 days for "PEG-PLA/CWO/PTX", 28 days for "PBS + X-Ray", 37 days for "PEG-PLA/CWO + X-Ray", and 45 days for "PEG-PLA/CWO/PTX + X-Ray".

Table 5. Results of the statistical analysis of - that is, p-values for-the mouse survival data presented in Figure 7 $(\mathrm{N}=8)$.

\begin{tabular}{|l|l|l|l|l|l|l|}
\hline & PBS & $\begin{array}{l}\text { PEG- } \\
\text { PLA/CWO }\end{array}$ & $\begin{array}{l}\text { PEG- } \\
\text { PLA/CWO/PTX }\end{array}$ & $\begin{array}{l}\text { PBS + } \\
\text { X-Ray }\end{array}$ & $\begin{array}{l}\text { PEG- } \\
\text { PLA/CWO + } \\
\text { X-Ray }\end{array}$ & $\begin{array}{l}\text { PEG- } \\
\text { PLA/CWO/PTX + } \\
\text { X-Ray }\end{array}$ \\
\hline PBS & - & 0.344 & 0.622 & 0.119 & 0.008 & 0.00002 \\
\hline
\end{tabular}




\begin{tabular}{|l|l|l|l|l|l|l|} 
PEG-PLA/CWO & - & - & 0.554 & 0.284 & 0.04 & 0.00005 \\
\hline $\begin{array}{l}\text { PEG- } \\
\text { PLA/CWO/PTX }\end{array}$ & - & - & - & 0.255 & 0.029 & 0.0001 \\
\hline PBS + X-Ray & - & - & - & - & 0.045 & 0.0007 \\
\hline $\begin{array}{l}\text { PEG-PLA/CWO + } \\
\text { X-Ray }\end{array}$ & - & - & - & - & - & 0.00008 \\
\hline
\end{tabular}

As shown in Figure 6, 8 Gy radiation caused a significant decrease in tumor growth, which is obvious, for instance, from comparison of the "PBS + X-Ray" data to the "PBS" data. Most importantly, a concomitant treatment with PEG-PLA/CWO/PTX NPs produced a significant enhancement of the tumor suppressive effect of X-rays (Figure 6). As shown in Figure 7, even without PTX, X-rays with concomitant PEG-PLA/CWO NPs caused a significant increase in survival time relative to X-ray radiation only, because of the CWO's radiosensitization effect [40] even though the synergistic effect of radiation and PEGPLA/CWO/PTX was not immediately visible in the tumor size study (Figure 6). PEGPLA/CWO/PTX NPs plus X-rays further increased the mouse survival by about 10 days relative to the "PEG-PLA/CWO + X-Ray" treatment. Log-rank analysis confirmed that the survival benefit produced by "PEG-PLA/CWO/PTX + X-Ray" is statistically significant relative to any other treatment: "PEG-PLA/CWO + X-Ray" ( $p=0.00008)$, "PBS + X-Ray" (p = 0.0007), "PEGPLA/CWO/PTX" ( $p=0.0001)$, "PEG-PLA/CWO" ( $p=0.00005)$, and "PBS" ( $=0.00002)$. It should be noted that in the absence of radiation, "PEG-PLA/CWO/PTX" behaved indistinguishably to "PEG-PLA/CWO" (Figure 7), which is consistent with the data shown in Figure 2(A) (which showed that PTX release from "PEG-PLA/CWO/PTX" is minimal in the absence of X-rays). Overall, these results clearly support the therapeutic potential of the concurrent X-ray and "PEG-PLA/CWO/PTX" therapy.

The results presented in Figures 6 and 7 strongly support that PEG-PLA/CWO/PTX NPs will produce significant effects of enhancing radiation effect and prolonging survival when using clinically relevant doses of radiation (i.e., $30-40$ fractions of $2-3$ Gy daily X-ray dose). In the present study, a sub-therapeutic radiation dose (4 fractions of $2 \mathrm{~Gy}$ per fraction given one fraction per day) was used in order to make the study in a mouse HN31 xenograft model manageable in terms of experiment timescale (less than a few months). However, data obtained using $4 \times 2$ Gy radiation (Figures 6 and 7), in fact, allow us to predict what will happen when usual clinical doses of radiation are used (as discussed below). 
Let us first discuss whether in vivo data shown in Figure 7 are predictable from in vitro data shown in Figure 5. Using the linear-quadratic model parameters ( $\alpha$ and $\beta$ ) obtained from clonogenic assay results shown in Figure 5, we can predict values of survival fraction (SF) for HN31 cells irradiated with 8 Gy in 4 fractions; that is, $\operatorname{SF}(D=8$ Gy in 4 fractions $) \approx[S F(D=2$ Gy in single fraction) $]^{4}$, assuming that the time interval between radiation fractions (1 day) was sufficient for cell's recovery from sub-lethal radiation damage [57]. The resulting predicted values were: $\mathrm{SF}(\mathrm{D}=8 \mathrm{~Gy}$ in 4 fractions $) \approx 0.138$ for "Control", 0.0638 for "PEG-PLA/CWO", and 0.0172 for "PEG-PLA/CWO/PTX" (notations same as in Figure 5). In in vivo studies presented in Figures 6 and 7, HN31 xenografts were treated with X-rays when the tumor volume reached a level of about $150 \mathrm{~mm}^{3}$ (containing an estimated amount of about $1.5 \times 10^{8}$ cells assuming a cell density of $10^{9}$ cells per cc of tumor [55]). Therefore, in our in vivo studies, the number of clonogenically active cells within the tumor immediately following 4 fractions of 2 Gy radiation (at Day 4 in Figure 6) is estimated to be: $2.08 \times 10^{7}$ cells for "PBS + X-Ray", 9.57 $\times 10^{6}$ cells for "PEG-PLA/CWO + X-Ray", and $2.58 \times 10^{6}$ cells for "PEG-PLA/CWO/PTX + XRay". Separately, from Figure 6 (i.e., from the slope of the tumor growth curve for "PBS" between Days 4 and 12 in Figure 6), the doubling time of HN31 cells in mouse xenografts in vivo is estimated to be about 5.09 days. Now, using these information, the time it takes for the irradiated tumor to re-grow to a size of $2.0 \mathrm{cc}$ (which is the tumor volume limit used as a euthanasia criterion in our vivo studies) post $4 \times 2$ Gy radiation is estimated to be: 34 days post radiation for "PBS + X-Ray", 40 days post radiation for "PEG-PLA/CWO + X-Ray", and 49 days post radiation for "PEG-PLA/CWO/PTX + X-Ray". These predicted values of mouse survival time are very close to experimental values: as stated in the figure caption for Figure 7 , the median mouse survival times were measured to be 28 days post first NP administration (24 days post radiation) for "PBS + X-Ray", 37 days post first NP administration (33 days post radiation) for "PEG-PLA/CWO + X-Ray", and 45 days post first NP administration (41 days post radiation) for "PEG-PLA/CWO/PTX + X-Ray". Given the simplicity of the model used in the calculation and also other euthanasia criteria used in experiment (e.g., > 20\% weight loss), this agreement is quite surprising. Nevertheless, this analysis validates the consistency of data presented in Figures 5, 6 and 7.

Using the above SF model, now we can further predict mouse survival times under dose conditions close to clinical practice. For instance, if 20 fractions of 2 Gy per fraction are used, 
the survival fractions of $\mathrm{HN} 31$ cells are predicted to be: $\mathrm{SF}(\mathrm{D}=40$ Gy in 20 fractions $) \approx 5.12 \times$ $10^{-5}$ for "Control", $1.06 \times 10^{-7}$ for "PEG-PLA/CWO", and $1.50 \times 10^{-9}$ for "PEG-

PLA/CWO/PTX”. Using the same procedure as above, the time it takes for the irradiated tumor to re-grow to a size of $2.0 \mathrm{cc}$ (mouse survival time) post $20 \times 2$ Gy radiation is estimated to be: 92 days post radiation for "PBS + X-Ray", 120 days post radiation for "PEG-PLA/CWO + XRay", and 168 days post radiation for "PEG-PLA/CWO/PTX + X-Ray". Concurrent "PEGPLA/CWO/PTX + X-Ray" is, therefore, predicted to produce a significant survival benefit of about 3.5 months relative to X-rays only ("PBS + X-Ray") or about a month even compared to "PEG-PLA/CWO + X-Ray".

We note that it is not possible at this point to draw a definitive conclusion from this study as to whether these nanoparticles will indeed enhance X-ray's efficacy in treating real patients with spontaneous tumors under clinical radiation conditions. We are in the process of initiating a pilot clinical study to evaluate the efficacy of intratumorally injected PEG-PLA/CWO/PTX NPmediated concomitant chemo-radio combination therapy in veterninary patients with spontaneous tumors.

\subsection{Biodistribution of PEG-PLA/CWO/PTX NPs in tumor-bearing mice following intratumoral administration}

An ideal intratumoral NP drug carrier system would be the one that is sufficiently large to stay within the tumor boundary and at the same time) small enough to spread out uniformly throughout inside the tumor tissue. In this regards, the previous literature [58] suggests that the ideal NP size should be about $30-60 \mathrm{~nm}$. The present PEG-PLA/CWO/PTX NP formulation was developed based on these considerations. A biodistribution (BD) study was performed to evaluate whether PEG-PLA/CWO/PTX NPs stay localized at the solid tumor site for the duration of a normal course of radiation therapy (40 - 50 days for HNSCC [37]) following intratumoral administration in the HN31 xenograft mouse model. A long tumor residence time of PEGPLA/CWO/PTX NPs (> one month) will enable a single injection of these nanopartciels at the beginning of treatment period to replace multiple daily/weekly injections of standard chemo radio-sensitizers [59]. Complete retention of NPs within the infused tumor region is also key to controlling the PTX availability within the tumor and minimizing systemic side effects. In this 
study, 21 mice were divided into 7 groups of 3 mice each (6 treatment groups, and one control group). All mice in treatment groups received an identical treatment, i.e., an intratumoral injection of PEG-PLA/CWO/PTX NPs (to a final NP concentration of $10 \mathrm{mg}$ CWO per cc tumor, injected in 2 portions at $\mathrm{t}=0$ and 1 days) following by X-ray radiation (320 keV, 4 fractions of 2 Gy per day over 4 days, i.e., at Days 1,2, 3 and 4); the treatment details were the same as in the efficacy study discussed above (Section 3.5). The control group was treated with vehicle (PBS) only (with no radiation therapy) and sacrificed at Day 2. Animals in different groups were euthanized at different time points $(\mathrm{t}): \mathrm{t}=2$ day (Group I, exposed to 2 Gy radiation on Day 1); $\mathrm{t}$ $=4$ days (Group II, exposed to $2+2$ Gy radiation on Days 1 and 2 , respectively); $\mathrm{t}=6$ days (Group III, exposed to $2+2+2$ Gy radiation on Days 1, 2 and 3, respectively); $\mathrm{t}=8$ days (Group IV, exposed to $2+2+2+2$ Gy radiation on Days 1, 2, 3 and 4, respectively); $\mathrm{t}=16$ days (Group V, exposed to $2+2+2+2$ Gy radiation on Days 1, 2, 3 and 4, respectively); and $\mathrm{t}$ $=31$ days (Group VI, exposed to $2+2+2+2$ Gy radiation on Days 1, 2, 3 and 4, respectively)). Tumor, blood and organ (brain, heart, kidney, lung, liver and spleen) samples were collected, and analyzed for calcium (Ca) content by atomic absorption spectroscopy (AAS) [36] (N = 3). The results of this BD study are presented in Figure 8. As shown in the figure, the data confirmed that the CWO NPs remained localized in the tumor for (at least) 31 days after injection. Over this one-month measurement period, intratumoral CWO NP retention was maintained at a level between about $70 \%$ and $90 \%$ with statistical fluctuations due to measurement uncertainties $(\mathrm{N}=$ 3); note that, even at one day after the completion of NP injections ("Day 2"), the amount of CWO NPs detected in the tumor appears to have been significantly less than the amount injected, which is thought to be due to loss of the NP solution during the intratumoral injection process. Trace amounts of CWO NPs were occasionally detected in other organs, but these results were statistically insignificant $(\mathrm{N}=3)$. 


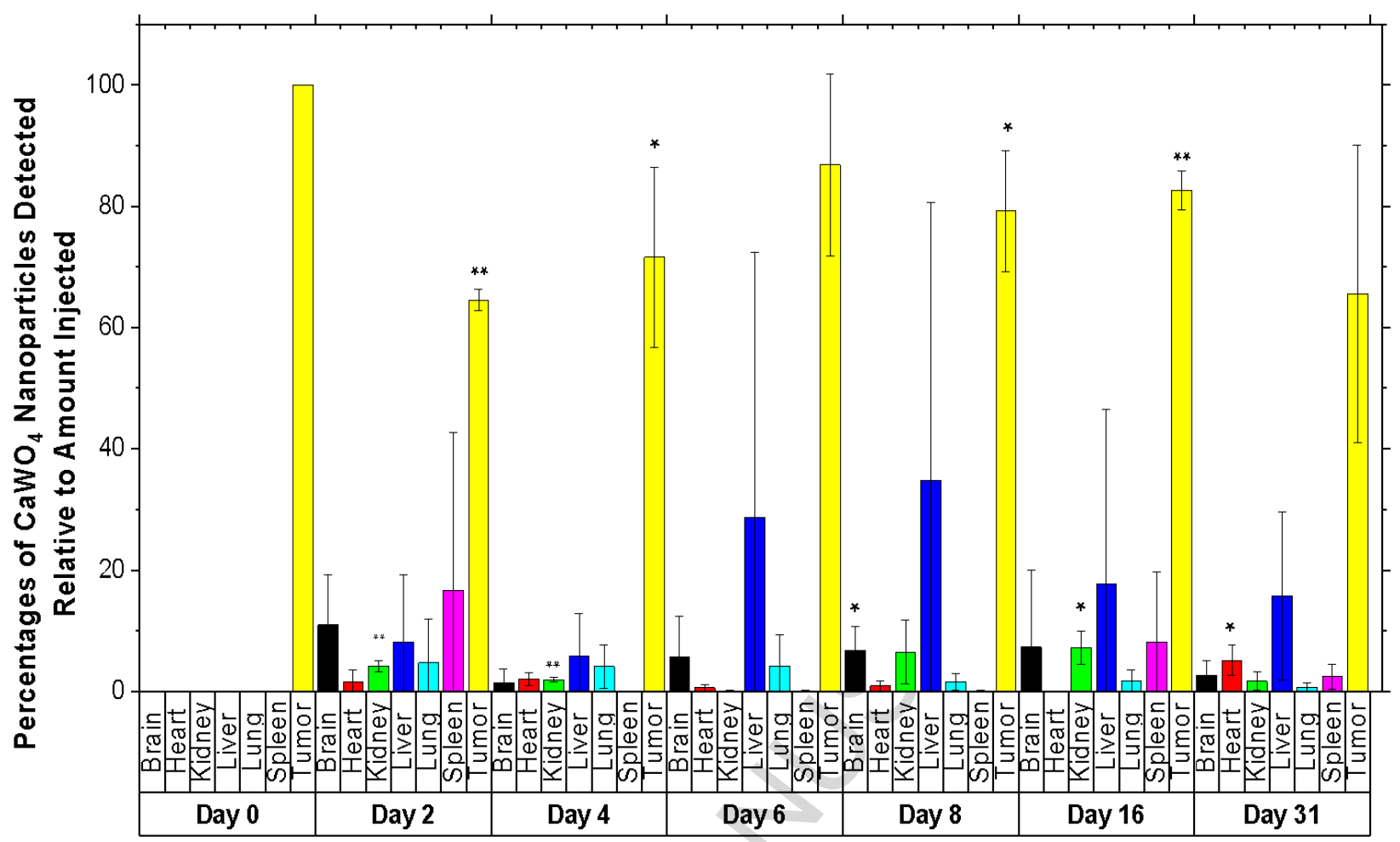

Figure 8. Time-dependent BD profiles of PEG-PLA/CWO/PTX NPs in tumor and major organs determined by AAS following intratumoral administration in HN31 mouse xenografts. Error bars represent standard deviations (N = 3). In control mice, no CWO was detected in any organs (data not shown). The "Day 0" bar graphs represent theoretical CWO NP BD levels that would be obtained immediately after NP injection. In some measurement groups (particularly, at Days 6 and 8), the sum of the percentage injected doses was greater than 100 percent because of errors associated with AAS measurements. Statistical analysis was performed for all organs and times relative to "Day 0" using one-way ANOVA. A difference is statistically significant if $\mathrm{p}<0.05\left(^{*}\right)$. A difference is statistically highly significant if $\mathrm{p}<0.005\left(^{* *}\right)$. The $\mathrm{p}$-values for all measurement groups are summarized in Table 6 below.

Table 6. Results of the statistical analysis of - that is, p-values for - the CWO NP BD data presented in Figure 8 (N $=3$ ).

\begin{tabular}{|l|l|l|l|l|l|l|}
\hline & Day 2 & Day 4 & Day 6 & Day 8 & Day 16 & Day 31 \\
\hline Brain & 0.08 & 0.699 & 0.213 & 0.04 & 0.374 & 0.118 \\
\hline Heart & 0.244 & 0.027 & 0.117 & 0.126 & 1 & 0.021 \\
\hline Kidney & 0.001 & 0.001 & 0.3739 & 0.091 & 0.01 & 0.123 \\
\hline Liver & 0.273 & 0.228 & 0.318 & 0.259 & 0.347 & 0.122 \\
\hline Lung & 0.323 & 0.118 & 0.228 & 0.117 & 0.2 & 0.116 \\
\hline Spleen & 0.327 & 1 & 0.124 & 0.373 & 0.285 & 0.116 \\
\hline Tumor & $3.84 \mathrm{E}-06$ & 0.029 & 0.2 & 0.022 & $7.31 \mathrm{E}-04$ & 0.0716 \\
\hline
\end{tabular}




\subsection{Multi-compartmental model for predicting in vivo pharmacokinetics (PK) of intratumorally injected PTX}

In order to gauge the clinical benefits of using PTX-loaded PEG-PLA-encapsulated CWO NPs ("PEG-PLA/CWO/PTX" NPs) over conventional PTX formulations, PK modeling was performed using a simple multi-compartment kinetic model. Clinical PEG-PLA/CWO/PTXbased intratumoral chemo-radio combination therapy would involve two steps: (1) intratumoral injection of PEG-PLA/CWO/PTX NPs, and (2) X-ray irradiation of the nanoparticle-treated tumor. The dynamics of intratumoral PTX concentration can be modeled with reasonable fidelity using a simplistic multi-compartmental PK model. Key kinetic processes involved can be summarized as follows. Radiation triggers the release of PTX from the polymer coating layer inside the tumor; in the absence of radiation, the PTX release is very slow. Released PTX will accumulate in the tumor compartment. On the other hand, there is continuous loss of PTX to the tumor exterior (e.g., by diffusion). The PTX eliminated from the tumor mainly enters the cardiovascular circulatory system, and eventually becomes cleared from the body through the kidneys $[60,61]$. A diagrammatic summary of these concepts is also presented in Scheme 2 below. 


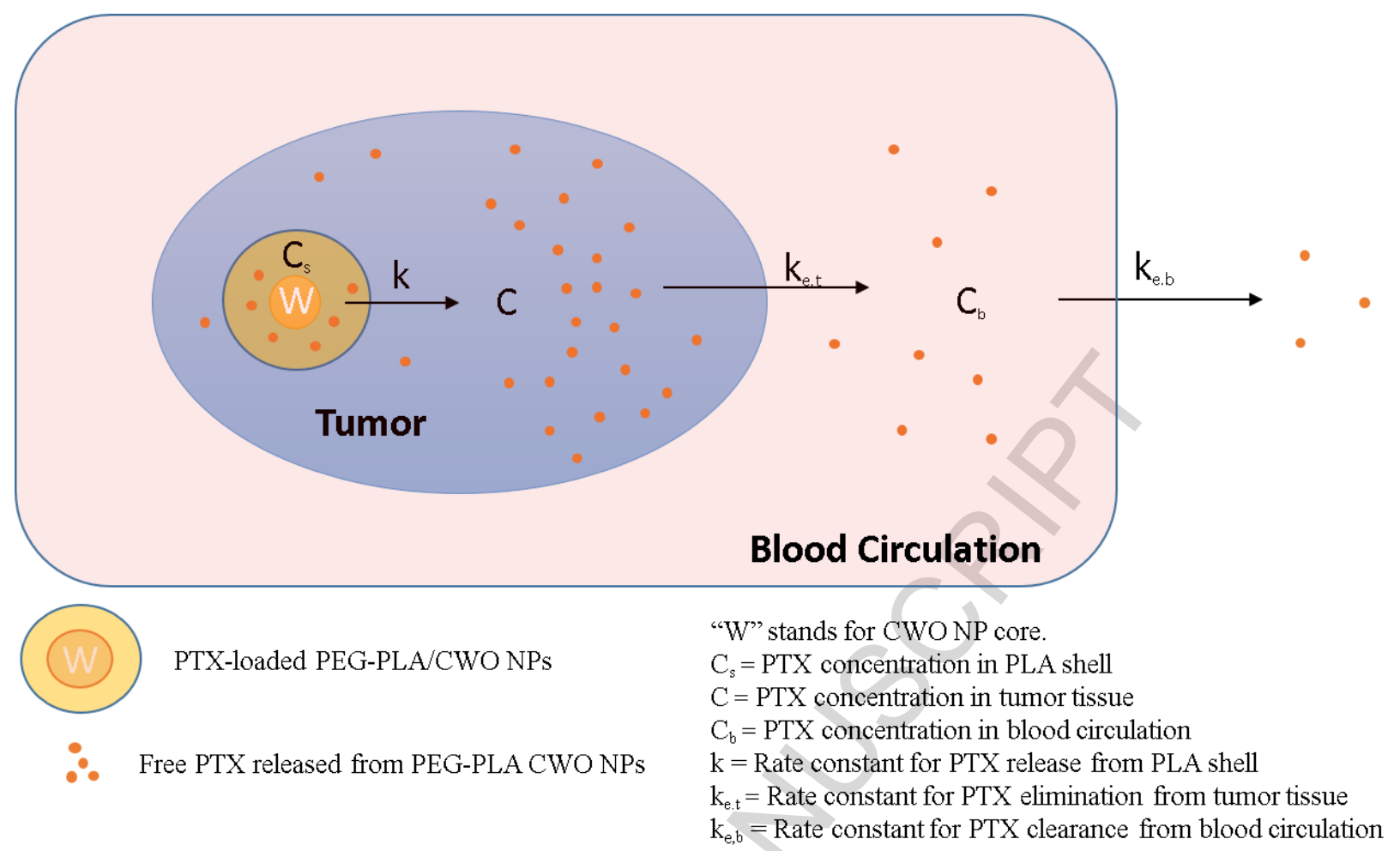

Scheme 2. Schematic description of the multi-compartmental pharmacokinetic model used in this study to estimate PTX concentrations in the PEG-PLA/CWO NPs, the tumor tissue, and the blood circulation system (denoted as $\mathrm{C}_{\mathrm{s}}$, $\mathrm{C}$, and $\mathrm{C}_{\mathrm{b}}$, respectively) as functions of time following intratumoral administration of PEG-PLA/CWO/PTX NPs.

In clinics, patients with locally advanced head and neck squamous cell carcinomas (HNSCC) typically undergo radiotherapy at a total radiation dose of $66-74 \mathrm{~Gy}$. The protocol is that the total dose is distributed over a period of $40-50$ days in 2 Gy daily fractions ( 5 fractions per week on week days with rest on weekends) [37]. PTX PK simulations were performed under this exact same radiation dose setting. The values of the rate constants describing the respective rates of PTX release from PEG-PLA/CWO/PTX NPs in the absence and presence of 2 Gy X-ray radiation were estimated from the experimental data shown in Figure 2(A). It was assumed that the solid tumor had a volume of $100 \mathrm{cc}$ (assumed to be invariant over time), and the tumor was initially injected with three different doses of PEG-PLA/CWO/PTX NPs (2, 5 or $10 \mathrm{mg}$ CWO per cc of tumor); of note, the tumor size actually did not influence PTX PK calculations, but the initial nanoparticle dose level, of course, did influence PTX PK calculations. The initial PTX concentration in the PLA coating layer was fixed at $20 \%$ by weight for all calculations. The Xray dose used was $70 \mathrm{~Gy}$, divided into 2 Gy daily fractions (with 5 fractions per week and rest on weekends as in clinical practice). The intraparticle, intratumoral and intracirculatory PTX PK profiles were traced for 210 days ( $\approx 7$ months); all radiation sessions were completed by Day 47 , 
and no radiation was given in the remaining period. Previously, the tumor elimination rate constant for PTX intratumorally delivered to mouse MCF7 xenografts in the polymer encapsulated form has been reported: $\mathrm{k}_{\mathrm{e}, \mathrm{t}} \approx 0.005 \mathrm{~h}^{-1}$ [62]. A slightly lower tumor PTX elimination constant value $\left(\mathrm{k}_{\mathrm{e}, \mathrm{t}} \approx 0.001 \mathrm{~h}^{-1}\right)$ was assumed for PEG-PLA/CWO/PTX NPs and PEG-PLA/PTX micelles assuming that spontaneous (human) tumors have a denser tissue structure. The intratumoral PTX concentration was calculated as a function of time by solving the following differential kinetic equation, ([rate of PTX accumulation within tumor] = [rate of PTX release from nanoparticles] - [rate of PTX elimination from tumor (e.g., due to diffusion to surrounding tissue, metabolization, etc.)]):

$$
\frac{\mathrm{dC}}{\mathrm{dt}}=\mathrm{k}\left(\mathrm{C}_{\mathrm{s}}-\mathrm{C}\right)-\mathrm{k}_{\mathrm{e}, \mathrm{t}} \mathrm{C}
$$

where $\mathrm{C}$ is the PTX concentration within the tumor (in Molar units), $\mathrm{C}_{\mathrm{s}}$ is the PTX concentration within the PLA "shell" layer (in Molar units), $\mathrm{k}$ is the rate constant for PTX release from the PLA layer $\left(\mathrm{k} \approx 1.3 \times 10^{-3}\right.$ and $4.3 \times 10^{-3} \mathrm{~h}^{-1}$, respectively, for unradiated and 2-Gy-irradiated situations, estimated from data shown in Figure 1(A) (i.e., from the slopes of the PTX release profiles at times between 2 and 3 days)), and $k_{e, t}$ is the rate constant for PTX elimination from the tumor $\left(\mathrm{h}^{-1}\right)$ (value taken from the literature as mentioned above). Note that from the data shown in Figure 2(A), the rate constant for PTX release under 7 Gy radiation is estimated to be $\mathrm{k}$ $\approx 1.6 \times 10^{-2} \mathrm{~h}^{-1}$, which suggests that the radiation-triggered PTX release rate is roughly linearly proportional to radiation dose. The initial condition used was: $\mathrm{C}=0$ at $\mathrm{t}=0 . \mathrm{C}_{\mathrm{s}}$ is coupled to $\mathrm{C}$ by the mass balance:

$$
\mathrm{C}_{\mathrm{s}} \mathrm{V}_{\mathrm{s}}=\mathrm{C}_{\mathrm{s}, \mathrm{o}} \mathrm{V}_{\mathrm{s}, \mathrm{o}}-\int_{0}^{\mathrm{t}} \mathrm{k}\left(\mathrm{C}_{\mathrm{s}}-\mathrm{C}\right) \mathrm{Vdt}
$$

where $\mathrm{V}_{\mathrm{s}}$ is the total volume of the PLA layers within the tumor, $\mathrm{V}$ is the volume of tumor, $\mathrm{C}_{\mathrm{s}, \mathrm{o}}=$ $\mathrm{C}_{\mathrm{s}}(\mathrm{t}=0)$, and $\mathrm{V}_{\mathrm{s}, \mathrm{o}}=\mathrm{V}_{\mathrm{s}}(\mathrm{t}=0)$; for simplicity, it was assumed that $\mathrm{V}$ and $\mathrm{V}_{\mathrm{s}}$ did not change with time (i.e., $\mathrm{V}=100 \mathrm{cc}$, and $\mathrm{V}_{\mathrm{s}}=\mathrm{V}_{\mathrm{s}, \mathrm{o}}$ at all times); note that constant $\mathrm{V}_{\mathrm{s}}$ means that the integrity of the PLA layer is maintained at all times. Equation (2) was solved simultaneously with Equation (3) to obtain predictions for $\mathrm{C}$ and $\mathrm{C}_{\mathrm{s}}$ as functions of time.

Figure 9(A) displays the results of these computations for three different types of PTX formulation (PEG-PLA/CWO/PTX, PEG-PLA/PTX, and Taxol) under various initial nanoparticle/PTX dose conditions (0.2, 0.5 and $1.0 \mathrm{mg}$ PTX per cc tumor). Also shown as a 
guide is the in vitro $\mathrm{IC}_{90}$ value of PTX (i.e., PTX concentration giving rise to $90 \%$ cell kill in vitro $\approx 90 \mu \mathrm{g} / \mathrm{mL}[63]$ ) (horizontal dotted line). As shown in the figure, in the presence of $\mathrm{CaWO}_{4}$, radiation triggers PTX release, and the intratumoral PTX concentration showed an increasing trend during the initial phase of treatment involving radiation (i.e., for the first 47 days). This initial boost in PTX dose helped in prolonging the PTX availability within the tumor above the therapeutic threshold (e.g., $\mathrm{IC}_{90}$ ) throughout and beyond the radiotherapy session. Also, as can be seen from Figure 9(A), the tumor availability of intratumorally administered PTX was significantly influenced by the total initial amount of PTX injected. However, at an identical total amount of PTX injected, it was obvious that the PEG-PLA/CWO/PTX system was able to maintain the therapeutic PTX level for a much longer period of time (e.g., by > 20 days at 1 mg/cc PTX dose) than the PEG-PLA/PTX system.
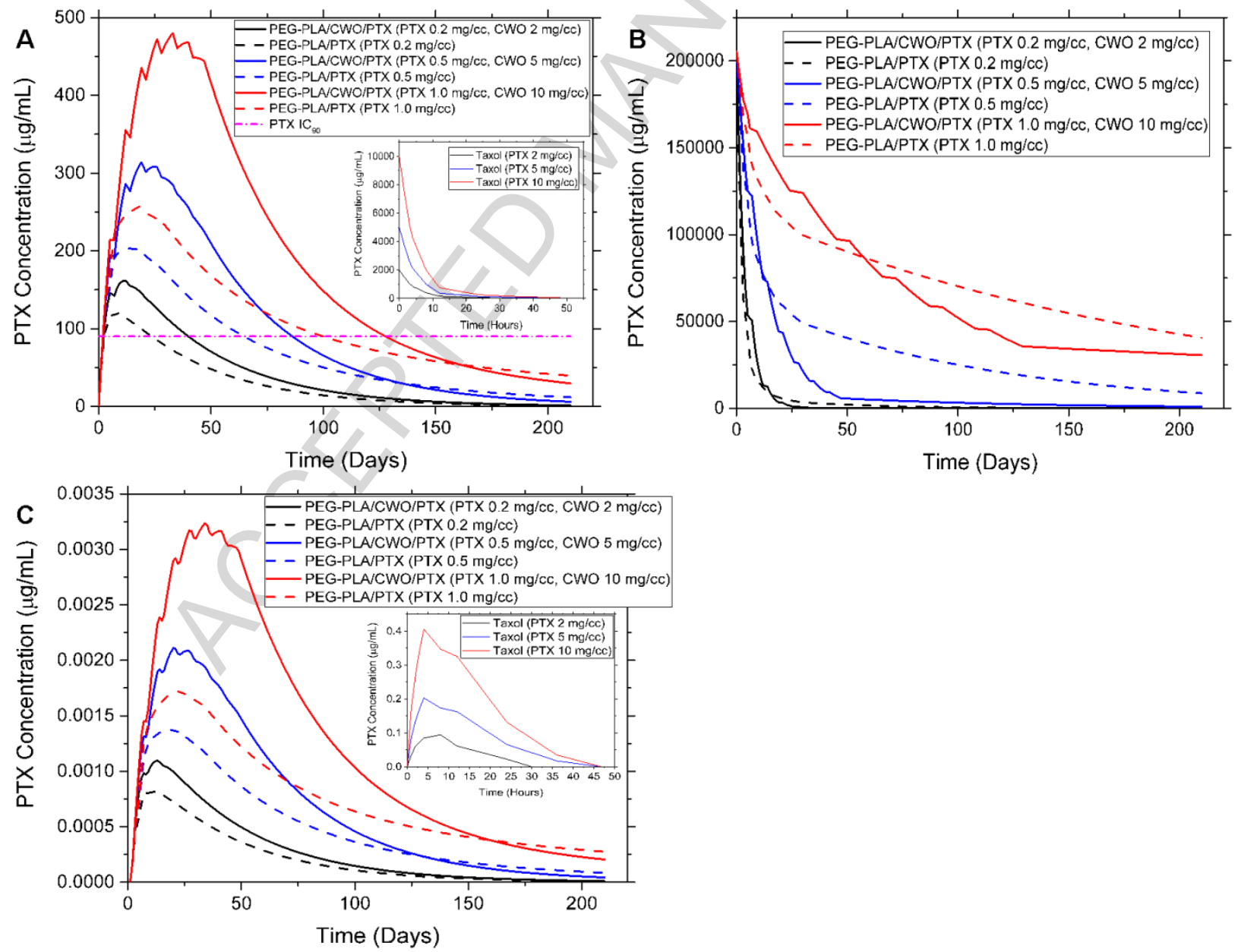
Figure 9. (A) Tumor concentrations of PTX released from PEG-PLA/CWO/PTX NPs (solid curves), PEGPLA/PTX micelles (dashed curves), and Taxol (PTX in an organic medium) (inset) estimated as functions of time following intratumoral injection of these respective formulations at 3 different PTX concentrations of 2,5 and 10 mg PTX per cc tumor. In these calculations, it was assumed that tumor received a total 70 Gy $320 \mathrm{keV} \mathrm{X-ray} \mathrm{dose} \mathrm{in}$ 35 fractions of daily 2 Gy radiation over an initial 47-day period; these daily fractions were given 5 days a week with a rest over the weekend similarly to real clinical practice. After Day 47, no further radiation was given. PTX release was monitored up to Day 210 . The dotted horizontal line represents the $\mathrm{IC}_{90}$ value of PTX measured in vitro in HN12 cells and thus the minimum PTX concentration for therapeutic efficacy. In the presence of CWO NPs, PTX release was controlled by X-ray irradiation, which enabled sustained release of PTX over a more extended period of time. Intratumorally injected Taxol exhibited extremely high PTX concentrations for few hours initially, but PTX was completely cleared from the tumor within 36 hours. (B) PTX concentrations in the PLA shell layers of PEGPLA/CWO/PTX NPs (solid curves) and PEG-PLA/PTX micelles (dashed curves) calculated as functions of time. PTX dose and X-ray radiation parameters used in these calculations were the same as in Figure 9(A). (C) PTX concentrations in blood circulation following intratumoral administration of PEG-PLA/CWO/PTX NPs (solid curves), PEG-PLA/PTX micelles (dashed curves), and Taxol (PTX in an organic medium) (inset). It was simplistically assumed that PTX released from tumor enters the blood circulation, and is eventually cleared from the body, e.g., through the kidney and/or liver. Blood concentrations of PTX released from PEG-PLA/CWO/PTX NPs and PEG-PLA/PTX micelles were several orders of magnitude lower than the maximum tolerable systemic dose of PTX in humans. Intratumoral administration of Taxol exhibited significantly higher blood PTX concentrations for a few hours initially. For all calculations, the tumor volume $(V)$ was assumed to be constant/unchanging $(=100 \mathrm{cc})$.

As shown in Figure 2(B), the PTX-loaded PEG-PLA micelle system exhibited an initial burst release; the drug release rate was very high initially (between Days 0 and 10), dropped rapidly afterward, and became stagnant for the rest of the period; the PEG-PLA/PTX system released about half of the loaded PTX within the first 10 days. Although "burst release" has positive aspects (immediate therapeutic effects, easier to overcome drug resistance, etc.), it is generally considered a downside because it is difficult to avoid even when such effect is not desired [64]. In the presence of co-encapsulated CWO NPs (i.e., in the PEG-PLA/CWO/PTX system), the initial burst PTX release phase was not observed (Figure 2(A)). Instead, radiation could be used to create a short period of rapid PTX release on demand in a highly controlled manner (e.g., > 50\% PTX released within a couple of days following 7 Gy radiation). In the PEG-PLA/CWO/PTX case, PTX release can be externally controlled by radiation; radiation dose and frequency influence PTX release. As demonstrated in Figure 2(A), this radiation-controlled PTX release mechanism enabled to maintain PTX tumor levels in the therapeutic range for a longer period (e.g., for > 120 days at $1 \mathrm{mg} / \mathrm{cc}$ PTX dose). PTX intratumorally delivered in the form of Taxol remained in the tumor, for instance, for $<12$ hours at a PTX dose of $10 \mathrm{mg} / \mathrm{cc}$.

Figure 9(B) displays how the PTX concentration in the PLA layer of a PEGPLA/CWO/PTX or PEG-PLA/PTX nanoparticle decreased with time. It was observed that in the PEG-PLA/PTX case, the PTX concentration in the PLA layer dropped rapidly in the initial 
"burst release" phase ( 0 - 10 days), followed by a second phase of much slower PTX release. In the PEG-PLA/CWO/PTX case, radiation enabled to extend the period of rapid release to about 50 days; about $70 \%$ of initially loaded PTX was released from the PLA layer during this rapid release (i.e., radiotherapy) period. As a consequence, the tumor PTX concentration was maintained at therapeutic levels for a longer period of time (Figure 9(A)).

It is reasonable to expect that after leaving the tumor, PTX will be mainly absorbed by the (blood) circulatory system. It is useful to estimate the PTX concentration in the circulatory system; high levels of PTX in the blood could produce systemic toxicity. The PTX concentration in the blood can be calculated using the mass balance equation

$$
\frac{\mathrm{d}\left(\mathrm{C}_{\mathrm{b}} \mathrm{V}_{\mathrm{b}}\right)}{\mathrm{dt}}=\mathrm{k}_{\mathrm{e} . \mathrm{t}} \mathrm{CV}-\mathrm{k}_{\mathrm{e} . \mathrm{b}} \mathrm{C}_{\mathrm{b}} \mathrm{V}_{\mathrm{b}}
$$

where $C_{b}$ is the PTX concentration in the blood, $V_{b}$ is the total blood volume in humans $(\approx 4700$ $\mathrm{mL}$ in a healthy adult human male [65]), and $\mathrm{k}_{\mathrm{e} . \mathrm{b}}$ is the rate constant for PTX renal clearance in humans $\left(\approx 0.336 \pm 0.002 \mathrm{~h}^{-1}[66]\right)$. The results of simulations for three different types of PTX formulation (PEG-PLA/CWO/PTX, PEG-PLA/PTX, and Taxol) under various initial nanoparticle/PTX dose conditions (0.2, 0.5 and $1.0 \mathrm{mg}$ PTX per cc tumor) are presented in Figure 9(C). At an identical initial PTX dose, the PTX concentration in the blood for the PEGPLA/CWO/PTX system was higher than that for the PEG-PLA/PTX system because of the higher PTX concentration in the tumor in the former situation (Figure 9(A)). A typical PTX dose in systemic chemotherapy is about $200 \mathrm{mg} / \mathrm{m}^{2}$ in humans [37], which translates into a value of about 100 in the units of $\mu \mathrm{g}$ PTX per mL blood (based on the blood volume of $4700 \mathrm{ml}$ for a healthy adult human male [65]); this PTX dose level causes dermatological side effects (in skin, hair, nail, etc.) in $86.8 \%$ of the patients treated, and cognitive/mental health-related problems in $75.0 \%$ of the patients treated $[67,68]$. The blood concentration of PTX intratumorally administered using the PEG-PLA/CWO/PTX (or PEG-PLA/PTX) delivery system was several orders of magnitude below this toxic threshold, which, therefore, supports that the intratumoral chemo-radio therapy proposed in this article will not, indeed, produce systemic chemo drug side effects. The blood concentration of PTX delivered in the form of Taxol peaked at a few hours post-administration and was significantly higher than PTX delivered using the PEGPLA/CWO/PTX or PEG-PLA/PTX formulation (inset of Figure 9(C)). 


\section{CONCLUSION}

The present study demonstrates radiation-controlled drug release nanoparticle formulations ("PEG-PLA/CWO/PTX NPs") as a means to achieve maximum bioavailability and minimum adverse effects of the chemo drugs (PTX), and also their ability to affect head and neck cancer cells (in vitro) and xenografts (in vivo). Specifically, the following conclusions can be drawn.

1. Using this novel PEG-PLA/CWO/PTX formulation, an uncontrolled initial burst release of PTX can be avoided (Figure 2). Further, radiation can be used to control the PTX availability within the tumor in a manner that has not previously been possible; radiation triggers the photo-lytic degradation of the PLA polymer and consequently the rapid release of PTX from nanoparticles (Figure 2). The PTX release kinetics can be adjusted by variation of, for instance, radiation dose (Figure 2).

2. An in vitro clonogenic survival study in human head and neck cancer HN31 cells suggests that concomitant PEG-PLA/CWO/PTX NPs significantly increase the biological cell killing effectiveness of X-rays. The SER value was estimated to be 1.40 for the PEGPLA/CWO/PTX-treated cells. PTX released from nanoparticles sensitizes HN31 cells to low doses of X-ray radiation (Figure 5).

3. No significant toxicity was observed in HN31 cultures treated with PEG-PLAencapsulated CWO NPs (Figure 4).

4. The efficacy of PTX-loaded PEG-PLA/CWO-based intratumoral chemo-radio therapy was evaluated in mouse HN31 tumor xenografts with sub-therapeutic doses of concurrent radiation. Concomitant PEG-PLA/CWO/PTX NPs significantly suppressed tumor growth (Figure 6) and also significantly enhanced mouse survival relative to X-rays only (Figure 7).

5. Following intratumoral administration of PEG-PLA/CWO/PTX NPs in HN31 mouse xenografts, time-dependent BD profiles of CWO NPs were determined in tumor and major organs (brain, heart, kidney, lung, liver, and spleen). CWO NPs remained localized in the tumor for (at least) 30 days (Figure 8).

6. A multi-compartmental PK model based on experimental rate constant data predicts that under the standard radiotherapy dose/fractionation scheme, intratumorally delivered PEG-PLA/CWO/PTX NPs maintained the tumor PTX concentration at therapeutic levels 
over a long period of time that covers the entire radiotherapy treatment period; therefore, only a single injection of nanoparticles at the beginning of the radiotherapy session is necessary (Figure 9). Also, this PK model demonstrates that the overall intratumoral and systemic PTX PK profiles can be adjusted by variations of such parameters as initial nanoparticle dose, and radiation dose/frequency. Future work is planned to experimentally validate these calculations.

This radiation-controlled drug release technology will enable patients with advanced solid tumors to achieve the benefits of chemo-radio combination treatment with reduced negative effects. This approach also presents a new therapeutic option that has not previously been available for pateints excluded from conventional chemo-radiotherapy protocols.

\section{Acknowledgements}

Funding for this research was provided by Purdue Office of the Executive Vice President for Research and Partnerships (OEVPRP) (New NIH R01 Program), Indiana Clinical and Translational Sciences Institute (CTSI) (Collaboration in Translational Research (CTR) Pilot Grant Program), Purdue University Center for Cancer Research (PCCR, P30CA023168) (SIRG Graduate Research Assistantship, Shared Resource Biological Evaluation Project, and Phase I Concept Award), Purdue University Discovery Park (Walther Oncology Physical Sciences \& Engineering Research Embedding Program), Lodos Theranostics LLC (Gift Grant), and the School of Chemical Engineering at Purdue University. The HN31 cell line was generously provided by Dr. Jeffrey N. Myers at the MD Anderson Cancer Center.

\section{Financial Conflict of Interest}

A company, Lodos Theranostics LLC, is currently attempting to commercialize the technology in this manuscript. The corresponding author, You-Yeon Won, has an ownership interest in this company. 


\section{REFERENCES}

[1] Head and Neck Cancer: Statistics., in: C.N.E.B.A.S.o.C.O. (ASCO). (Ed.), American Society of Clinical Oncology (ASCO), 2018.

[2] W. Li, Y. Yu, H. Wang, A. Yan, X. Jiang, Evaluation of the prognostic impact of postoperative adjuvant radiotherapy on head and neck mucosal melanoma: a meta-analysis, BMC Cancer, 15 (2015).

[3] G. Ku, D. Ilson, Role of Neoadjuvant Therapy for Esophageal Adenocarcinoma, Surgical Oncology Clinics of North America, 18 (2009) 533-+.

[4] S. Chauhan, J. Kaur, M. Kumar, A. Matta, G. Srivastava, A. Alyass, J. Assi, I. Leong, C. MacMillan, I. Witterick, T. Colgan, N. Shukla, A. Thakar, M. Sharma, K. Siu, P. Walfish, R. Ralhan, Prediction of recurrence-free survival using a protein expression-based risk classifier for head and neck cancer, Oncogenesis, 4 (2015).

[5] L. Serpe, Conventional Chemotherapeutic Drug Nanoparticles for Cancer Treatment, WileyVCH, 2007.

[6] A.A. Forastiere, H. Goepfert, M. Maor, T.F. Pajak, R. Weber, W. Morrison, B. Glisson, A. Trotti, J.A. Ridge, C. Chao, G. Peters, D.-J. Lee, A. Leaf, J. Ensley, J. Cooper, Concurrent Chemotherapy and Radiotherapy for Organ Preservation in Advanced Laryngeal Cancer, New England Journal of Medicine, 349 (2003) 2091-2098.

[7] F. Danhier, O. Feron, V. Preat, To exploit the tumor microenvironment: Passive and active tumor targeting of nanocarriers for anti-cancer drug delivery, Journal of Controlled Release, 148 (2010) 135-146.

[8] J.S. Ross, P.S. David, P. Robert, R. Mark, P.L. Gerald, S. James, E.S. Nancy, S.G. Geoffrey, S. W. Fraser, P. Lajos, N.H. Gabriel, Targeted Therapies for Cancer, American Journal of Clinical PathologyAmerican Journal of Clinical Pathology, 122122.4 (2004) 598-609.

[9] A.H. Partridge, B.H. J., W.E. P., Side Effects of Chemotherapy and Combined Chemohormonal Therapy in Women with Early-Stage Breast Cancer, JNCI MonographsJNCI Monographs, 30 (2001) 135-142.

[10] M.A.N. Sendur, S. Aksoy, Current Systemic Therapy Options for Head and Neck Cancers, Radiation Therapy for Head and Neck Cancers Radiation Therapy for Head and Neck Cancers (2014) 17-31.

[11] S. Kanotra, S. Kanotra, A. Gupta, J. Paul, Chemoradiation in Advanced Head and Neck Cancers: A Comparison of two Radiosensitizers, Paclitaxel and Cisplatin, Indian Journal of Otolaryngology and Head \&amp; Neck Surgery, 63 (2011) 229-236.

[12] L.J. Adkins D, Trinkaus K, Thorstad W, Lewis J Jr, Wildes T, Siegel BA, Dehdashti F, Gay H, Mehan P, Nussenbaum B., A Phase 2 Trial of Inductionnab-paclitaxel and Cetuximab given with Cisplatin and 5-fluorouracil Followed by Concurrent Cisplatin and Radiation for Locally Advanced Squamous Cell Carcinoma of the Head and Neck, Cancer 119 (2012) 766-773.

[13] D. Citrin, J. Mansueti, A. Likhacheva, L. Sciuto, P. Albert, S. Rudy, T. Cooley-Zgela, A. Cotrim, B. Solomon, A. Colevas, A. Russo, J. Morris, L. Herscher, S. Smith, C. Van Waes, Long-term outcomes and toxicity of concurrent paclitaxel and radiotherapy for locally advanced head-and-neck cancer, International Journal of Radiation Oncology Biology Physics, 74 (2009) 1040-1046.

[14] D. Givens, L. Karnell, A. Gupta, G. Clamon, N. Pagedar, K. Chang, D. Van Daele, G. Funk, Adverse Events Associated With Concurrent Chemoradiation Therapy in Patients With Head and Neck Cancer, Archives of Otolaryngology-Head \&amp; Neck Surgery, 135 (2009) 1209-1217. 
[15] A. Falchook, R. Green, M. Knowles, R. Amdur, W. Mendenhall, D. Hayes, J. GrilleyOlson, J. Weiss, B. Reeve, S. Mitchell, E. Basch, B. Chera, Comparison of Patient-and Practitioner-Reported Toxic Effects Associated With Chemoradiotherapy for Head and Neck Cancer, JAMA Otolaryngology-Head \&amp; Neck Surgery, 142 (2016) 517-523.

[16] A. Gupta, S. Baxi, C. Hoyne, Assessing feasibility, compliance and toxicity of concomitant chemo-radiotherapy in head and neck cancers in the Northern Territory: initial experience and challenges, J Med Radiat Sci, 64 (2017) 131-137.

[17] M. Hautmann, M. Hipp, O. Kolbl, Clostridium difficile-associated diarrhea in radiooncology: an underestimated problem for the feasibility of the radiooncological treatment?, Radiation Oncology, 6 (2011).

[18] B. Wenig, J. Werner, D. Castro, K. Sridhar, H. Garewal, W. Kehrl, A. Pluzanska, O. Arndt, P. Costantino, G. Mills, F. Dunphy, E. Orenberg, R. Leavitt, The role of intratumoral therapy with cisplatin/epinephrine injectable gel in the management of advanced squamous cell carcinoma of the head and neck, Archives of Otolaryngology-Head \&amp; Neck Surgery, 128 (2002) 880-885.

[19] M. Westphal, D. Hilt, E. Bortey, P. Delavault, R. Olivares, P. Warnke, I. Whittle, J. Jaaskelainen, Z. Ram, A phase 3 trial of local chemotherapy with biodegradable carmustine $(\mathrm{BCNU})$ wafers (Gliadel wafers) in patients with primary malignant glioma, Neuro-Oncology, 5 (2003) 79-88.

[20] E.P. Goldberg, Hadba, A. R., Almond, B. A., Marotta, J. S., Intratumoral cancer chemotherapy and immunotherapy: opportunities for nonsystemic preoperative drug delivery, Journal of Pharmacy and Pharmacology, 54 (2010) 159-180.

[21] B.D. Weinberg, E. Blanco, J. Gao, Polymer Implants for Intratumoral Drug Delivery and Cancer Therapy, Journal of Pharmaceutical Sciences, 97 (2008) 1681-1702.

[22] J.C. Bateman, H.N. Carlton, PALLIATION OF MAMMARY CARCINOMA WITH PHOSPHORAMIDE DRUGS, Journal of the American Medical Association, 162 (1956) 701706.

[23] W. Hohenforst-Schmidt, P. Zarogoulidis, K. Darwiche, T. Vogl, E.P. Goldberg, H. Huang, M. Simoff, Q. Li, R. Browning, F.J. Turner, P. Le Pivert, D. Spyratos, K. Zarogoulidis, S.I. Celikoglu, F. Celikoglu, J. Brachmann, Intratumoral chemotherapy for lung cancer: re-challenge current targeted therapies, Drug Des Devel Ther, 7 (2013) 571-583.

[24] L. Miao, S. Guo, C.M. Lin, Q. Liu, L. Huang, Nanoformulations for combination or cascade anticancer therapy, Adv Drug Deliv Rev, 115 (2017) 3-22.

[25] P.G. H. Brem, Biodegradable polymer implants to treat brain tumors, Journal of Controlled Release, 74 (2001) 63 - 67.

[26] G.K. C. Le Pechoux, E. Deutsch, P. Sargos, A. Levy, T. De Baere, X. Buy, F. Martinetti, E. Stoeckle, P. Terrier, A. Le Cesne, A. Italiano, J.C. Soria and S. Bonvalot, PD-0045: Ph I/II study evaluating the impact of nanoparticules combined to pre-operative radiotherapy in soft tissue sarcoma, in, Radiotherapy and Oncology, 2015, pp. Pages S21-S22.

[27] M.G. Ewend, J.A. Williams, K. Tabassi, B.M. Tyler, K.M. Babel, R.C. Anderson, M.L. Pinn, D.J. Brat, H. Brem, Local delivery of chemotherapy and concurrent external beam radiotherapy prolongs survival in metastatic brain tumor models, Cancer Research, 56 (1996) 5217-5223.

[28] M.G. Ewend, P. Sampath, J.A. Williams, B.M. Tyler, H. Brem, Local delivery of chemotherapy prolongs survival in experimental brain metastases from breast carcinoma, Neurosurgery, 43 (1998) 1185-1192. 
[29] S. Kakinoki, T. Taguchi, H. Saito, J. Tanaka, T. Tateishi, Injectable in situ forming drug delivery system for cancer chemotherapy using a novel tissue adhesive: Characterization and in vitro evaluation, European Journal of Pharmaceutics and Biopharmaceutics, 66 (2007) 383-390. [30] J. Kim, J. Lee, K. Kim, K. Na, S. Song, J. Lee, H. Kuh, Intratumoral delivery of paclitaxel using a thermosensitive hydrogel in human tumor xenografts, Archives of Pharmacal Research, 36 (2013) 94-101.

[31] P. Ma, J.R. Mumper, Paclitaxel Nano-Delivery Systems: A Comprehensive Review, Journal of Nanomedicine \& Nanotechnology Journal of Nanomedicine \&amp; Nanotechnology 4(2013) 1-35.

[32] M. Nikl, Scintillation detectors for x-rays, Measurement Science and Technology, 17 (2006) R37-R54.

[33] S.D. Jo, J. Lee, M.K. Joo, V.J. Pizzuti, N.J. Sherck, S. Choi, B.S. Lee, S.H. Yeom, S.Y. Kim, S.H. Kim, I.C. Kwon, Y.Y. Won, PEG-PLA-Coated and Uncoated Radio-Luminescent CaWO4 Micro- and Nanoparticles for Concomitant Radiation and UV-A/Radio-Enhancement Cancer Treatments, Acs Biomaterials Science \& Engineering, 4 (2018) 1445-1462.

[34] A.f.T.S.a.D. Registry, A Toxicological Profile for Tungsten, U.S. Department of Health and Human Services, 2003.

[35] J. Lee, N. Rancilio, J. Poulson, Y. Won, Block Copolymer-Encapsulated CaWO4 Nanoparticles: Synthesis, Formulation, and Characterization, Acs Applied Materials \& Interfaces, 8 (2016) 8608-8619.

[36] J. Lee, S. Choi, K. Kim, H. Heng, S. Torregrosa-Allen, B. Ramsey, B. Elzey, Y. Won, Nontoxic Formulations of Scintillation Nanocrystals for Use as X-ray Computed Tomography Contrast Agents, Bioconjugate Chemistry, 28 (2017) 171-182.

[37] M.M. Stevenson, Head and Neck Cancer Treatment Protocols: Treatment Protocols, in: J.G. Petruzelli (Ed.), 2016.

[38] W.-W. Weng, Y.-J. Xu, J.-M. Wan, Z.-Z. Wang, W.-X. Ding, M. Liu, C.-J. Hong, Molecular Mechanism of Radiosensitizing Effect of Paclitaxel, Chinese Journal of Cancer Chinese Journal of Cancer, 28 (2009) 844-850.

[39] J. Hiro, Y. Inoue, Y. Toiyama, S. Yoshiyama, K. Tanaka, Y. Mohri, C. Miki, M. Kusunoki, Possibility of paclitaxel as an alternative radiosensitizer to 5-fluorouracil for colon cancer, Oncology Reports, 24 (2010) 1029-1034.

[40] J.L. Sung Duk Jo, Min Kyung Joo, Vincenzo J. Pizzuti,Nicholas J. Sherck, Slgi Choi, Beom Suk Lee, Sung Ho Yeom, Sang Yoon Kim, Sun Hwa Kim, Ick Chan Kwon, You-Yeon Won, PEG-PLA-Coated and Uncoated Radio-Luminescent CaWO4 Micro- and Nanoparticles for Concomitant Radiation and UV-A/Radio-Enhancement Cancer Treatments, ACS Biomaterials Science \& Engineering, 4 (2018) 1445-1462.

[41] H.T. Chen, S.W. Kim, L. Li, S.Y. Wang, K. Park, J.X. Cheng, Release of hydrophobic molecules from polymer micelles into cell membranes revealed by Forster resonance energy transfer imaging, Proceedings of the National Academy of Sciences of the United States of America, 105 (2008) 6596-6601.

[42] D.S. Mei Zhao, Curtis R. Pickering, Samar A. Jasser, Ying C. Henderson, Gary L. Clayman, Erich M. Sturgis, Thomas J. Ow, Reuben Lotan, Thomas E. Carey, Peter G. Sacks, Jennifer R. Grandis, David Sidransky, Nils Erik Heldin, and Jeffrey N. Myers, Assembly and initial characterization of a panel of 85 genomically validated cell lines from diverse head and neck tumor sites, Clinical cancer research : an official journal of the American Association for Cancer Research, 17 (2011) 7248-7264. 
[43] H. Tada, O. Shiho, K. Kuroshima, M. Koyama, K. Tsukamoto, An improved colorimetric assay for Interleukin-2, Journal of Immunological Methods, 93 (1986) 157-165.

[44] S. Bonvalot, C. Le Pechoux, T. De Baere, G. Kantor, X. Buy, E. Stoeckle, P. Terrier, P. Sargos, J.M. Coindre, N. Lassau, R.A. Sarkouh, M. Dimitriu, E. Borghi, L. Levy, E. Deutsch, J.C. Soria, First-in-Human Study Testing a New Radioenhancer Using Nanoparticles (NBTXR3) Activated by Radiation Therapy in Patients with Locally Advanced Soft Tissue Sarcomas, Clinical Cancer Research, 23 (2017) 908-917.

[45] G.L.B. Andreia Araújo, Manuela Silva and Ana Vera Machado., UV Stability of Poly(Lactic Acid) Nanocomposites, Journal of Materials Science and Engineering B 3(2013) 7583.

[46] S.R. Catherine Zhang, Julie Goddard, Kara Constantine, Peter Collins, The Effect of UV Treatment on the Degradation of Compostable Polylactic Acid, Journal of Emerging Investigators 1(2013) 1-6.

[47] C.B. AlainCopineta, Stephanie Govindina, Veronique Coma, YvesCouturiera Effects of ultraviolet light $(315 \mathrm{~nm})$, temperature and relative humidity on the degradation of polylactic acid plastic films., Chemosphere 55 (2004) 763-773.

[48] R.H. Emad Yousif, Photodegradation and photostabilization of polymers, especially polystyrene: review. , Springer plus., 2 (2013) 398.

[49] R.M. Vincenzo J. Pizzuti, Jaewon Lee, Sandra E. Torregrosa-Allen, Melanie P.Currie,Scott R. Clark, Anish P. Patel, Christopher R. Schorr, Yava Jones-Hall, Michael O.Childress, Jeannie M. Plantenga , Nicholas J. Rancilio , Bennett D. Elzey ,You-YeonWon, Folic Acid-Conjugated Radio-Luminescent Calcium Tungstate Nanoparticles as Radio-Sensitizers for Cancer Radiotherapy, in: Nanomedicine, 2018

[50] J. Coronado, Fresno, F., Hernández-Alonso, M.D., Portela, R. , Design of Advanced Photocatalytic Materials for Energy and Environmental Applications, 2013.

[51] G.H. Yew, A.M.M. Yusof, Z.A.M. Ishak, U.S. Ishiaku, Water absorption and enzymatic degradation of poly(lactic acid)/rice starch composites, Polymer Degradation and Stability, 90 (2005) 488-500.

[52] J. Cadet, J.R. Wagner, DNA Base Damage by Reactive Oxygen Species, Oxidizing Agents, and UV Radiation, Cold Spring Harbor Perspectives in Biology, 5 (2013).

[53] J.A. Reisz, N. Bansal, J. Qian, W.L. Zhao, C.M. Furdui, Effects of Ionizing Radiation on Biological Molecules-Mechanisms of Damage and Emerging Methods of Detection, Antioxidants \& Redox Signaling, 21 (2014) 260-292.

[54] T. Konno, J. Watanabe, K. Ishihara, Enhanced solubility of paclitaxel using water-soluble and biocompatible 2-methacryloyloxyethyl phosphorylcholine polymers, Journal of Biomedical Materials Research Part a, 65A (2003) 209-214.

[55] U. Del Monte, Does the cell number 10(9) still really fit one gram of tumor tissue?, Cell Cycle, 8 (2009) 505-506.

[56] R.J. Mundt AJ, Chung TD, et al., Biologic Basis of Radiation Therapy, 6th ed., Hamilton (ON), 2003.

[57] E.J. Hall, A.J. Giaccia, Radiobiology for the Radiologist, 7th ed., Lippincott, Williams \& Wilkins, 2011.

[58] R.K. Jain, T. Stylianopoulos, Delivering nanomedicine to solid tumors, Nature Reviews Clinical Oncology, 7 (2010) 653-664.

[59] R.B.D. Muhammad Wasif Saif, Fluoropyrimidines as Radiation Sensitizers, in: T. H. Choy (C) Humana Press Inc., NJ (Ed.) Chemoradiation in Cancer Therapy, 2010. 
[60] W. Xiao, J. Luo, T. Jain, J. Riggs, H. Tseng, P. Henderson, S. Cherry, D. Rowland, K. Lam, Biodistribution and pharmacokinetics of a telodendrimer micellar paclitaxel nanoformulation in a mouse xenograft model of ovarian cancer, International Journal of Nanomedicine, 7 (2012) 1587-1597.

[61] W. Wang , L. Deng, S. Xu , X. Zhao, N. Lv, G. Zhang, N. Gu, R. Hu, J. Zhang, J. Liu, A. Dong, A reconstituted "two into one" thermosensitive hydrogel system assembled by drugloaded amphiphilic copolymer nanoparticles for the local delivery of paclitaxel, Journal of Materials Chemistry B, 1 (2012) 552-563.

[62] L.E. van Vlerken, Z. Duan, S.R. Little, M.V. Seiden, M.M. Amiji, Biodistribution and pharmacokinetic analysis of paclitaxel and ceramide administered in multifunctional polymerblend nanoparticles in drug resistant breast cancer model, Molecular Pharmaceutics, 5 (2008) 516-526.

[63] M. Boulin, S. Guiu, B. Chauffert, S. Aho, J.P. Cercueil, F. Ghiringhelli, D. Krause, P. Fagnoni, P. Hillon, L. Bedenne, B. Guiu, Screening of anticancer drugs for chemoembolization of hepatocellular carcinoma, Anticancer Drugs, 22 (2011) 741-748.

[64] Q. Sun, M. Radosz, Y. Shen, Challenges in design of translational nanocarriers, Journal of Controlled Release, 164 (2012) 156-169.

[65] I. World Book, Blood, Chicago, 1998.

[66] J. Vasantha, G. Kannan, T. Goud, T. Palani, Vanitha, R, R. Anitha, P. JMM, Pharmacokinetic Evaluation of Paclitaxel in South Indian Cancer Patients: A Prospective Study, 3 (2011) 322-328.

[67] M. Hackbarth, N. Haas, C. Fotopoulou, W. Lichtenegger, J. Sehouli, Chemotherapyinduced dermatological toxicity: frequencies and impact on quality of life in women's cancers. Results of a prospective study, Supportive Care in Cancer, 16 (2008) 267-273.

[68] C. Nordqvist, Chemotherapy: Types, uses, and adverse effects, in: C. Chun (Ed.) Medical News Today, 2017. 


\section{Highlights}

- Drug-loaded radioluminescent nanoparticles enable radiation-controlled drug release

- Concurrent drug-loaded radioluminescent nanoparticles enhance radiotherapy efficacy

- This approach has potential to enhance the therapeutic index for chemo radiation 
Graphical abstract

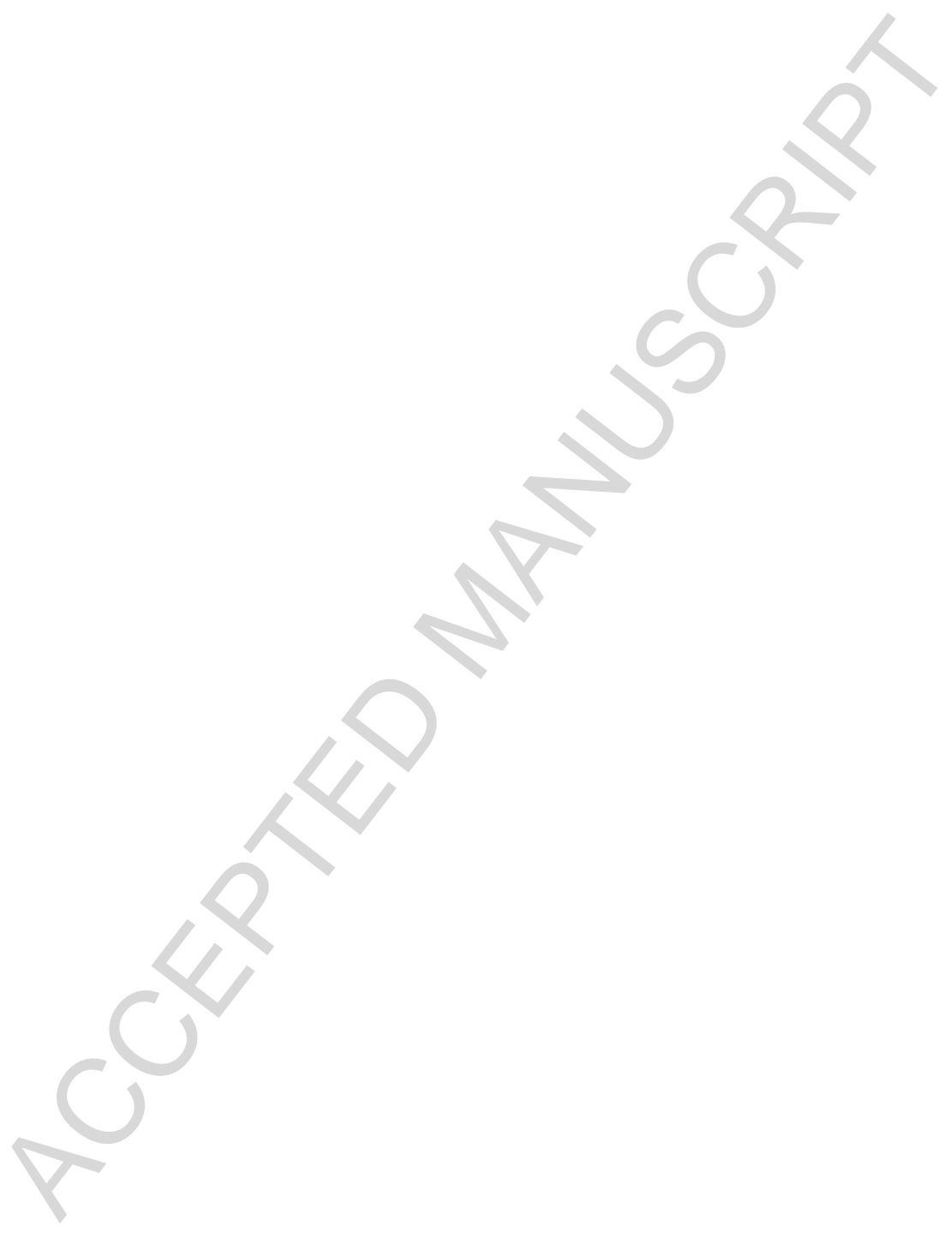

